03,08

\title{
Акцепторы III группы с мелкими и глубокими уровнями в карбиде кремния: исследования методами ЭПР и ДЭЯР
}

\author{
() И.В. Ильин, Ю.А. Успенская, Д.Д. Крамущенко, М.В. Музафарова, В.А. Солтамов, \\ Е.Н. Мохов, П.Г. Баранов
}

Физико-технический институт им. А.Ф. Иофрфе РАН,

Санкт-Петербург, Россия

๑ E-mail: pavel.baranov@mail.ioffe.ru

(Поступила в Редакцию 30 июня 2017 г.)

\begin{abstract}
Приведены результаты исследований акцепторов III группы (B, $\mathrm{Al}, \mathrm{Ga})$ в кристаллах карбида кремния с использованием наиболее информативных методов электронного парамагнитного резонанса и двойного электронно-ядерного резонанса и рассмотрены структурные модели акцепторов с мелкими уровнями и с глубокими уровнями. Наряду с данными, полученными ранее, добавлены исследования с использованием высокочастотных методов магнитного резонанса, позволивших обнаружить орторомбические отклонения от аксиальной симметрии для глубоких акцепторов, и проведен теоретический анализ, объясняющий наблюдаемые в экспериментах сдвиги $g$-факторов для глубоких акцепторов, обусловленные появлением орторомбических искажений, вероятно, вследствие наличия эффекта Яна-Теллера.
\end{abstract}

DOI: 10.21883/FTT.2018.04.45670.211

\section{1. Введение}

Элементы III группы: B, Al, Ga - являются наиболее важными акцепторными примесями в карбиде кремния $\mathrm{SiC}$. Эти примеси могут вводиться в кристалл как в процессе роста, так и путем диффузии или имплантации. Замещающий атом элемента III группы обычно является акцептором, так как у него не хватает одного валентного электрона для заполнения обычных тетраэдрических связей в $\mathrm{SiC}$. При относительно низких температурах дырка локализована вблизи акцептора, при высоких температурах акцептор может оказаться ионизованным, что приводит к $p$-типу проводимости. Акцепторы III группы в $\mathrm{SiC}$ исследовались методами емкостной спектроскопии глубоких уровней (DLTS), оптической спектроскопии, эффекта Холла [1]. Бор создает в запрещенной зоне $\mathrm{SiC}$ мелкие и глубокие уровни [1]. Центры бора с мелкими акцепторными уровнями (мелкий бор) с энергией активации $0.30-0.39 \mathrm{eV}$ обычно наблюдаются методами эффекта Холла и DLTS $[2,3]$. Второй тип центров бора с глубокими уровнями (глубокий бор) впервые наблюдался в экспериментах по фотолюминесценции $[4,5]$. Предполагалось, что характерная высокотемпературная желтая люминесценция $6 \mathrm{H}-\mathrm{SiC}: \mathrm{B}$ (наблюдающаяся до комнатной температуры) возникает из-за донорно-акцепторной рекомбинации между донорами азота и глубокими центрами бора. Соответственно, энергия ионизации глубоких центров бора оценивалась величиной более $0.65 \mathrm{eV}$. В исследованиях методом DLTS $p-n$-переходов, полученных диффузией бора [3], наблюдался глубокий центр с дублетной структурой $E_{V}+0.63 \mathrm{eV}$ и $E_{V}+0.73 \mathrm{eV}$. Только один глубокий уровень бора $E_{V}+0.58 \mathrm{eV}$ наблюдался в имплантированных бором эпитаксиальных слоях $6 H$-SiC [6]. Два акцепторных уровня, соответствующих мелкому и глубокому бору, наблюдались с помощью емкостной спектроскопии [7]. Из графиков Аррениуса и фотоемкостных измерений энергия активации центров была оценена величиной $0.22 \mathrm{eV}$ и $0.35 \mathrm{eV}$ для мелкого В и $0.55 \mathrm{eV}$ и $0.75 \mathrm{eV}$ для глубокого В.

Мелкий алюминий образует в запрещенной зоне $6 H$-SiC акцепторные уровни $E_{V}+0.239 \mathrm{eV}$ для гексагональной $(h)$ позиции в решетке и $E_{V}+0.2485 \mathrm{eV}$ для двух квазикубических позиций $(k 1, k 2)$; мелкий галлий создает акцепторные уровни $E_{V}+0.317 \mathrm{eV}(h)$ и $E_{V}+0.333 \mathrm{eV}(k 1, k 2)$ [8]. Энергетические уровни акцепторов III группы, найденные экспериментально в $6 H-\mathrm{SiC}$, показаны на рис. $1, a$. Энергия ионизации акцепторов III группы слабо зависит от политипа и от положения дефекта в решетке, так как максимум валентной зоны локализован в Г-точке и в результате отсутствуют эффекты интерференции. Напротив, энергия ионизации доноров $(\mathrm{N}, \mathrm{P})$ гораздо более чувствительна к политипу и положению примеси в решетке, что объясняется эффектами интерференции волновой функции донорного электрона, которая состоит из волновых функций нескольких минимумов зоны проводимости.

\section{2. Экспериментальные результаты}

2.1. Акцепторы с мелкими уровнями.

Спектры электронного парамагнитного резонанса (ЭПР) акцепторов с мелкими уровнями (мелкие акцепторы) в кубических полупроводниках (например, в кремнии, $\mathrm{Si}$ ), как правило, наблюдаются при приложении аксиального давления для снятия четырехкратного вырождения основного состояния акцептора, которое очень чувствительно к случайным напряжениям в кристалле. В некубических политипах карбида кремния (например, 

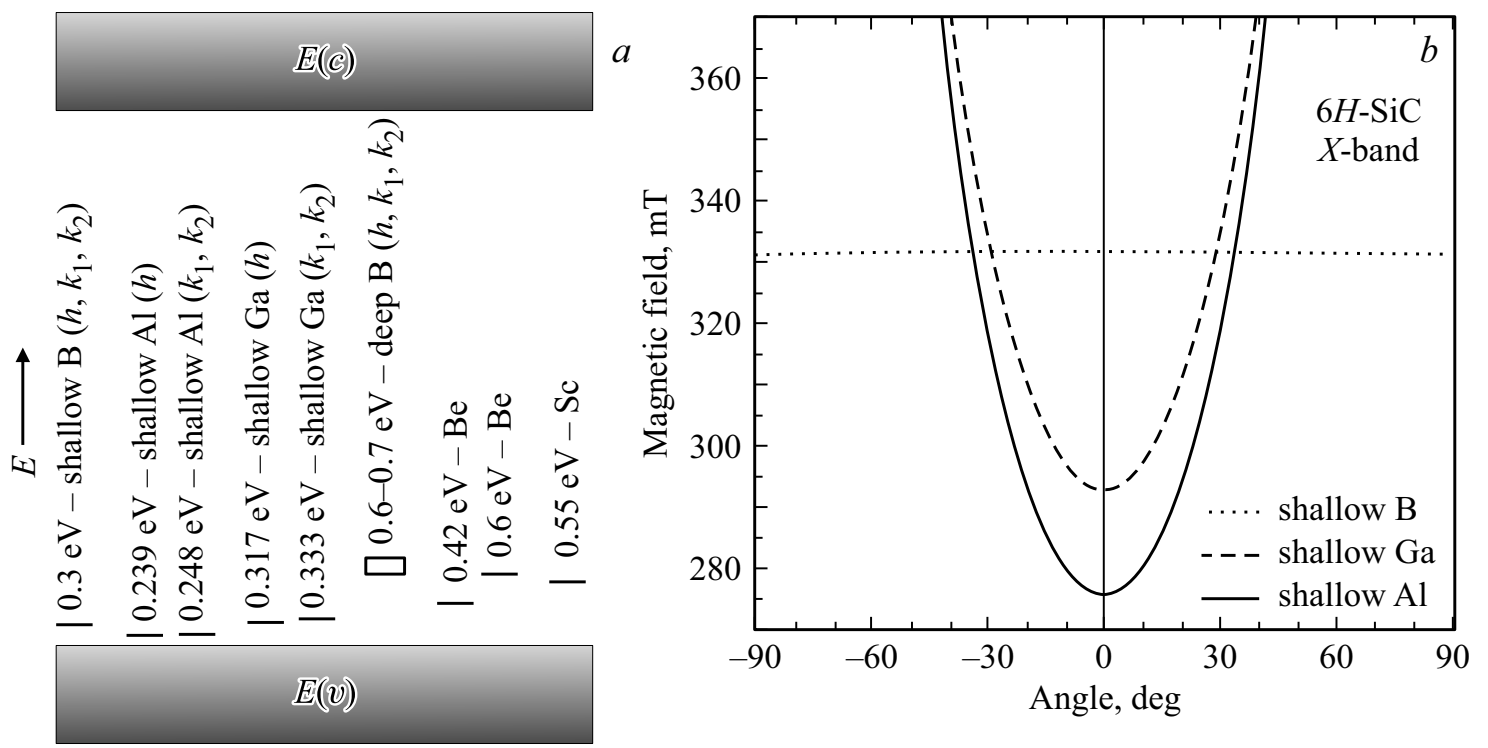

Рис. 1. (a) Схема акцепторных уровней, наблюдающихся в запрещенной зоне $6 H$-SiC. (b) Угловые зависимости сигналов ЭПР мелких акцепторов III группы в $X$-диапазоне в $6 H$-SiC. Представлены данные только для $h$-позиций.

6H-SiC) это вырождение уже снято кристаллическим полем, и спектры ЭПР акцепторов могут наблюдаться без приложения аксиального давления.

Первые наблюдения ЭПР акцепторов в $\mathrm{SiC}$ были произведены Woodbury и Ludwig [9], которые исследовали спектры ЭПР мелкого бора в $6 \mathrm{H}$-SiC. C тех пор примеси III группы, B, Al и Ga с мелкими уровнями в $\mathrm{SiC}$ изучались методами ЭПР [10-11], оптически детектируемого магнитного резонанса (ОДМР) [18-22], импульсного ЭПР и импульсного двойного электронноядерного резонанса (ДЭЯР) [23-25], а также методом ДЭЯР в непрерывном режиме (cw-ENDOR) [26-31].

Важный результат этих исследований - коренное отличие поведения мелких акцепторов бора по сравнению с другими акцепторами III группы, Al и Ga. Резонансные свойства мелких акцепторов $\mathrm{Al}$ и Ga главным образом определяются зонной структурой кристаллической решетки. В работах [16,20-23] было показано, что для этих акцепторов может быть использовано приближение эффективной массы, описывающее дырку в верхнем состоянии валентной зоны (состояние Г9), но с уменьшенным вкладом орбитального момента из-за локализации дырки. В отличие от $\mathrm{Al}$ и $\mathrm{Ga}$, поведение спектров ЭПР мелких акцепторов бора не описывается в приближении эффективной массы, более того, ЭПР мелкого бора, в отличие от мелких акцепторов $\mathrm{Al}$ и $\mathrm{Ga}$, может наблюдаться в кубическом политипе $3 C$ - $\mathrm{SiC}$ без приложения аксиального давления к кристаллу.

М елки й $\mathrm{B}$. Спектры ЭПР мелкого бора изучались при температурах $1.2-300 \mathrm{~K}$ в политипах карбида кремния $3 C$-, $4 H$ - и $6 H$-, легированных бором $[9,10,12,23]$. Бор имеет два стабильных изотопа: ${ }^{10} \mathrm{~B}$ (естественная распространенность $19.8 \%$, ядерный спин $I=3$ ) и ${ }^{11} \mathrm{~B}(80.2 \%, I=3 / 2)$. Отношение ядерных $g$-факторов
$g_{\mathrm{I}}\left({ }^{11} \mathrm{~B}\right) / g_{\mathrm{I}}\left({ }^{10} \mathrm{~B}\right)=3$, где $g_{\mathrm{I}}\left({ }^{11} \mathrm{~B}\right)$ и $g_{\mathrm{I}}\left({ }^{10} \mathrm{~B}\right)$ - ядерные $g$-факторы изотопов ${ }^{11} \mathrm{~B}$ и ${ }^{10} \mathrm{~B}$ соответственно. Спектры ЭПР мелких акцепторов ${ }^{11} \mathrm{~B}$ должны состоять из трех наборов по четыре линии, которые принадлежат трем кристаллографически неэквивалентным позициям в решетке $6 H$-SiC: гексагональной $(h)$ и двум квазикубическим $(k 1, k 2)$ и могут быть описаны обычным спиновым гамильтонианом. Для мелкого бора в $h$-позиции главная ось $g$-тензора параллельна оси $c$ кристалла, поэтому этот центр будем называть „аксиальный“ бор. Для мелкого бора в $k 1$ (или $k 2$ ) позиции главная ось $g$-тензора $z$ лежит в плоскости симметрии $\{11 \overline{2} 0\}$ кристалла и эта ось параллельна одному из неаксиальных направлений связи $\mathrm{Si}-\mathrm{C}$ (таких направлений шесть, так как в структуре вюрцита есть два типа тетраэдров). Мы будем обозначать соответствующие дефекты как „неаксиальный“ бор. Таким образом, $h$-позиция соответствует парамагнитному центру с симметрией $C_{3 v}$, квазикубические позиции ( $k 1$ или $k 2)$ соответствуют центрам с симметрией $C_{S}$.

Значения $g$-тензоров вдоль главных осей были измерены в различных политипах $\mathrm{SiC}[10,23]: g_{z}\left(g_{\|}\right)=$ $=2.0020( \pm 0.0002)$ и $g_{x}, g_{y}\left(g_{\perp}\right)=2.006( \pm 0.0005)$, где локальная ось $z$ соответствует одному из направлений $\mathrm{Si}-\mathrm{C}$ (для позиции $h$ это ось $c$ кристалла, для $k 1$ или $k 2$ - одно из шести неэквивалентных направлений $\mathrm{Si}-\mathrm{C}$, не совпадающих с осью $c$ ). Для кубического политипа $3 C$ - $\mathrm{SiC}$ - это одно из направлений $\langle 111\rangle$ кристалла. Оси $x$ и $y$ лежат в плоскости, перпендикулярной оси $z$, при этом ось $x$ лежит в одной из плоскостей $\{11 \overline{2} 0\}$ в $6 H-\mathrm{SiC}(4 H-\mathrm{SiC})$. Таким образом, $g$-тензор мелких центров бора практически аксиален относительно локальной оси $z$ для всех мелких акцепторов бора в кубических и гексагональных политипах $\mathrm{SiC}$. Показано, 
что $g$-фактор вдоль оси $z$ практически равен $g$-фактору спина свободного электрона. Существует небольшое влияние аксиального кристаллического поля вдоль оси $c$ в гексагональных политипах $\mathrm{SiC}$ для квазикубических позиций („неаксиальный“ дефект), но это эффект второго порядка. Отклонение от аксиальной симметрии для анизотропного $g$-тензора оценено величиной $\left|g_{x}-g_{y}\right|<0.0005$ для квазикубических узлов.

Тензор сверхтонкого $(\mathrm{CT})$ взаимодействия $A$ отражает аксиальную симметрию мелких центров бора вдоль локальной оси $z$. Для квазикубических позиций в гексагональных политипах $\mathrm{SiC}$ („неаксиальный“ дефект) наблюдается небольшое отклонение от аксиальной симметрии, которое отражает влияние кристаллического поля вдоль оси $c$ и не играет особой роли для понимания структуры мелких акцепторов бора. При $4.2 \mathrm{~K}$ для $3 C$-SiC: $A_{\|}= \pm 1.0 \cdot 10^{-4} \mathrm{~cm}^{-1}, A_{\perp}=(\mp) 1.9 \cdot 10^{-4} \mathrm{~cm}^{-1}$; для $6 H-\mathrm{SiC} A_{\|}= \pm 1.8 \cdot 10^{-4} \mathrm{~cm}^{-1}, A_{\perp}=(\mp) 1.3 \cdot 10^{-4} \mathrm{~cm}^{-1}$ ( $h$-узел); $A_{\|}= \pm 1.7 \cdot 10^{-4} \mathrm{~cm}^{-1}, A_{\perp}=(\mp) 0.9 \cdot 10^{-4} \mathrm{~cm}^{-1}$ (k1-узел); $A_{\|}= \pm 2.0 \cdot 10^{-4} \mathrm{~cm}^{-1}, A_{\perp}=(\mp) 1.1 \cdot 10^{-4} \mathrm{~cm}^{-1}$ $(k 2$-узел). Следует отметить, что контактное $a$ и дипольдипольное $b$ СТ взаимодействия имеют разные знаки $\left(A_{\|}=a+2 b, A_{\perp}=a-b\right)$. Это было показано измерениями ЭПР $[9,12]$ и позже подтверждено исследованиями ДЭЯР [20,22,25-31].

Когда магнитное поле параллельно оси $c$, наблюдаются дополнительные СТ линии. Их интенсивность примерно соответствует наличию изотопа ${ }^{29} \mathrm{Si} \mathrm{c} \mathrm{распростра-}$ ненностью $4.7 \%(I=1 / 2)$, равномерно распределенного по трем эквивалентным позициям вблизи узла, занимаемого акцептором В. Величина этого СТ взаимодействия с соседними ядрами $\mathrm{Si}$, которое часто называют суперсверхтонкое (ССТ) взаимодействие, практически изотропна $a \cong 9 \cdot 10^{-4} \mathrm{~cm}^{-1}$ для политипов $3 \mathrm{C}$-SiC, $6 H$-SiC и $4 \mathrm{H}$-SiC. Естественная распространенность изотопа ${ }^{13} \mathrm{C}$ составляет всего $1.1 \%(I=1 / 2)$ и ССТ структура, обусловленная углеродом, практически не наблюдается. Проводились измерения на образцах $6 \mathrm{H}-\mathrm{SiC}$, специально обогащенных изотопом ${ }^{13} \mathrm{C}$ до $30 \%[10,23]$. В этих образцах наблюдалось ССТ взаимодействие с одним атомом углерода для мелкого акцептора бора. Это взаимодействие измерялось в разных политипах и имеет величину $A_{\|}=(56-58) \cdot 10^{-4} \mathrm{~cm}^{-1}$ для магнитного поля, параллельного оси $z$ и $A_{\perp}=(15-17) \cdot 10^{-4} \mathrm{~cm}^{-1}$ для магнитного поля, направленного перпендикулярно оси z. Для мелкого акцептора бора, замещающего $\mathrm{Si}$, четыре соседних атома $\mathrm{C}$ должны быть эквивалентны, но результаты ЭПР показывают, что они не эквивалентны в отношении распределения спиновой плотности. Спектры ЭПР мелкого бора резко меняются при температурах выше $50 \mathrm{~K}[10,12]$. Происходит термически активированное перемещение по трем смещенным позициям для гексагональных позиций и по четырем позициям для мелкого бора в кубическом $\mathrm{SiC}$, что приводит к усреднению спектров в диапазоне высоких температур.

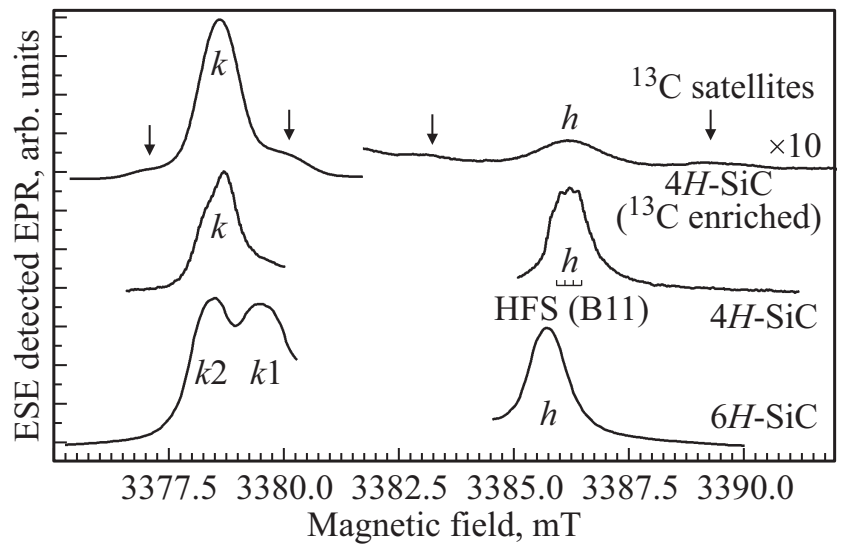

Рис. 2. (Верхний сигнал) Спектр ЭПР акцепторов мелкого бора в кристалле $4 H-\mathrm{SiC}$, обогащенном ${ }^{13} \mathrm{C}$, частота $95 \mathrm{GHz}$, ориентация $\mathbf{B} \| c$. Спектр состоит из двух линий: одна относится к атому В в квазикубической позиции $(k)$, вторая к атому В в гексагональной позиции $(h)$. Дополнительные сателлиты обусловлены СТ взаимодействием с ближайшим атомом ${ }^{13} \mathrm{C}$. (Средний сигнал) Спектр ЭПР акцепторов мелкого бора в $4 H$-SiC с естественной распространенностью ${ }^{13} \mathrm{C}$. (Нижний сигнал) Спектр ЭПР акцепторов мелкого бора в кристалле $6 H$-SiC в ориентации В $\| c$. Все спектры получены при $1.2 \mathrm{~K}$.

Исследования мелких акцепторов бора в образцах $4 H-\mathrm{SiC}$ и $6 H-\mathrm{SiC}$ с естественным распространением изотопов и в образцах, обогащенных ${ }^{13} \mathrm{C}$, проводились также с помощью высокочастотного $(95 \mathrm{GHz})$ импульсного ЭПР и ДЭЯР [23-25]. По СТ взаимодействию неспаренного электрона с ядрами ${ }^{13} \mathrm{C}(I=1 / 2)$ было установлено пространственное распределение электронной волновой функции. Показано, что есть небольшая разница в степени локализации этой волновой функции между разными узлами - квазикубическими и гексагональными - в политипах $4 H-\mathrm{SiC}$ и $6 H-\mathrm{SiC}$. В частности, показано, что пространственное распределение сильно анизотропно. Эта анизотропия может быть объяснена учетом анизотропии массы дырки, то есть предположением, что теория эффективной массы (частично) работает при описании удаленной части пространственного распределения электронной волновой функции.

На рис. 2 показаны три спектра ЭПР мелких акцепторов бора в $\mathrm{SiC}$ в диапазоне $95 \mathrm{GHz}$ при В $\| c$. Верхний спектр получен в кристалле $4 H-\mathrm{SiC}$, обогащенном ${ }^{13} \mathrm{C}$, и состоит из двух линий, одна из которых относится к атому бора в $k$-позиции, вторая - к атому бора в $h$-позиции. Низкая интенсивность сигнала от $h$-позиции может объясняться положением уровня Ферми. Слабые сателлиты в усиленном спектре возникают из-за СТ взаимодействия с соседним ядром ${ }^{13} \mathrm{C}$. Средний спектр получен в монокристалле $4 H$-SiC с естественным содержанием изотопов. Наблюдается также СТ взаимодействие с ядерным спином ${ }^{11} \mathrm{~B}$ для мелких акцепторов, 

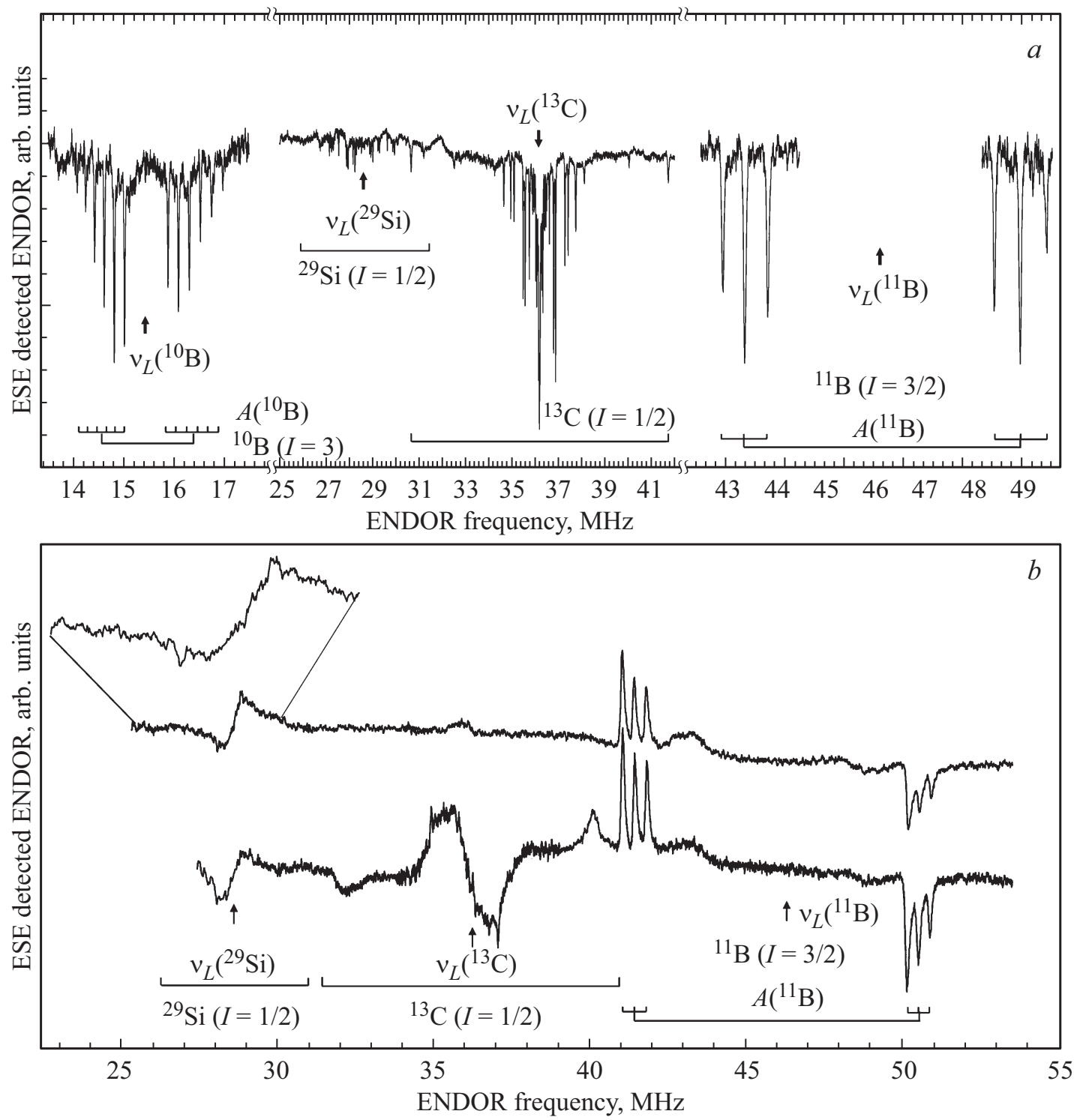

Рис. 3. (a) Спектр ДЭЯР, измеренный по электронному спиновому эху (ЭСЭ) мелкого бора в $k$-позиции в $6 H$-SiC на частоте $95 \mathrm{GHz}$, в ориентации $\mathbf{B} \| c$ при температуре $1.2 \mathrm{~K}$. Очевидно разделение линий ДЭЯР, принадлежащих разным изотопам и ядрам. Так как образец обогащен изотопом ${ }^{13} \mathrm{C}(30 \%)$, сигнал ДЭЯР от этого изотопа очень интенсивный. Только внешние линии ДЭЯР ${ }^{13} \mathrm{C}$ немного перекрываются с линиями ${ }^{29} \mathrm{Si}$ и ${ }^{11} \mathrm{~B}$. Стрелками обозначены ларморовские частоты соответствующих изотопов в полях, в которых измерялись сигналы ЭПР. (b) Спектры ДЭЯР, зарегистрированные по ЭСЭ глубокого бора в $6 H$-SiC с естественным содержанием изотопов (наверху) и обогащенном $(30 \%)$ изотопом ${ }^{13} \mathrm{C}$ (внизу) при $95 \mathrm{GHz}$, в ориентации В $\| c$ при температуре $1.2 \mathrm{~K}$. Линии, принадлежащие ${ }^{13} \mathrm{C}$, видны в спектре обогащенного образца. На вставке показан увеличенный сигнал вблизи зеемановской частоты ${ }^{29} \mathrm{Si}$.

находящихся в $h$-позиции. Нижний спектр получен в кристалле $6 \mathrm{H}$ - $\mathrm{SiC}$, обогащенном ${ }^{13} \mathrm{C}$ и легированном изотопом ${ }^{11} \mathrm{~B}$.

На рис. 3, $a$ показан общий вид спектра ДЭЯР мелких акцепторов, полученного в $6 H-\mathrm{Si}^{13} \mathrm{C}$ с естественным содержанием бора в ориентации В $\| c$ на частоте $95 \mathrm{GHz}$ при температуре $1.2 \mathrm{~K}$. Рисунок представляет отличный пример высокого спектрального разрешения сверхтонких взаимодействий для различных ядер, которое можно получить с использованием высокочастотного ДЭЯР.
Наиболее заметный результат изучения ДЭЯР возможность приписать линии ДЭЯР ${ }^{13} \mathrm{C}$ конкретному атому углерода на расстоянии до 11 длин связи от атома бора. Это позволило определить пространственное распределение спиновой плотности неспаренного электрона акцептора мелкого бора и, в частности, изучить разницу в электронных свойствах квазикубических и гексагональных узлов в $4 H-\mathrm{SiC}$ и $6 H-\mathrm{SiC}$ [24]. В качестве примера на рис. 4 мы приводим визуализацию делокализации электронной волновой функции, связанной с узлом $k 1$, на рисунке показана спиновая плотность 


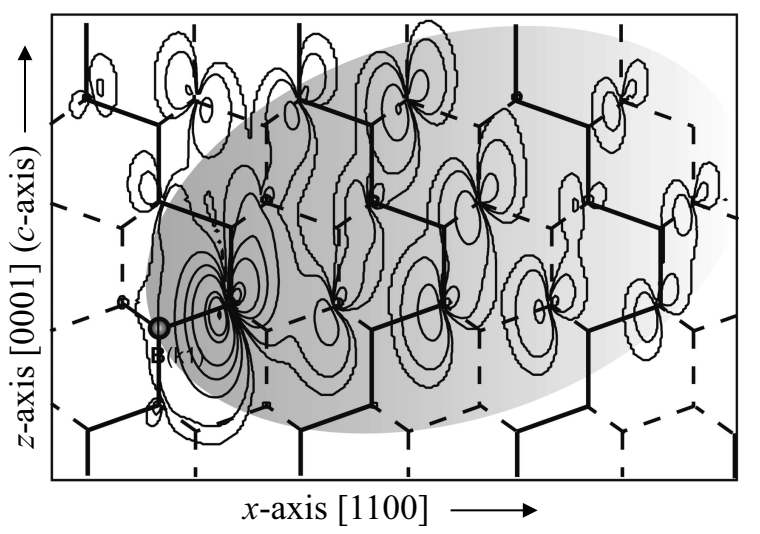

Рис. 4. Величина квадрата волновой функции неспаренного электрона мелкого бора в $6 H-\mathrm{SiC}$ для позиции $k 1$ в плоскости $\{11 \overline{2} 0\}$ показана как функция положения в плоскости $x z$ изолиниями. Указано положение ядра В. Положение атома С, несущего максимальную спиновую плотность, может быть определено по сгущению изолиний. „Лесенки“ сплошных линий показывают связи в решетке, расположенные в плоскости $\{11 \overline{2} 0\}$, содержащей атом В и атом C, несущий максимальную спиновую плотность (главная плоскость), штриховые линии показывают проекции связей, расположенных в плоскостях \pm 1 , на главную плоскость. Очевидно, что спиновая плотность является резко анизотропной. Распределение направлено от атома В только по одному направлению вдоль линии В-C под углом $70^{\circ}$ к оси $c$.

на атомах С в главной плоскости $\{11 \overline{2} 0\}$, то есть в плоскости, которая содержит ось $c$. Кристалл можно рассматривать как набор „лесенок“ вдоль оси $c$. Лесенка, в которой расположены атом В и основной атом $\mathrm{C}$, показана сплошной линией. Следующие лесенки расположены в плоскостях $\{11 \overline{2} 0\}$ на ступень выше и на ступень ниже, их проекции на плоскость, содержащую атом В и основной атом С, показаны штриховыми линиями. Видно, что электронная спиновая плотность распределена в виде эллипсоида с главной осью, расположенной под углом $70^{\circ}$ к оси $c$, то есть по направлению атом B-атом C с наибольшей спиновой плотностью.

Показано, что все три мелких акцептора бора в $6 H$-SiC имеют разную локализацию спиновой плотности и, таким образом, разные энергии ионизации. Возникает вопрос, может ли анизотропное распределение электронной волновой функции на удаленных ядрах ${ }^{13} \mathrm{C}$, полученное по данным ДЭЯР, быть объяснено в рамках теории эффективной массы. Мелкий галлий (shallow $\mathrm{Ga}-s \mathrm{Ga})$ и мелкий алюминий $(s \mathrm{Al})$, как будет показано ниже, в противоположность мелкому бору $(s \mathrm{~B})$ имеют сильно анизотропный $g$-тензор, в соответствии с расчетной анизотропией $g$-фактора дырки в валентной зоне. В случае акцептора $s$ В орбитальный момент заморожен и свойства зонной структуры не должны отражаться на свойствах дырки на акцепторе $s$ В. Тем не менее, электронная волновая функция акцептора sB в квазикубических позициях в плоскости, перпенди- кулярной оси $c$, делокализована значительно сильнее, чем в направлении, параллельном оси $c$ в соответствии с анизотропией эффективной массы $m_{h \|}=1.67 m_{e}$ и $m_{h \perp}=0.62 m_{e}$. Отметим, что анизотропия в перпендикулярной плоскости усредняется при высоких температурах, когда происходит перепрыгивание связи между бором и углеродом с наибольшей спиновой плотностью между тремя возможными направлениями этой связи. В этом случае спиновая плотность делокализована сильнее в перпендикулярной плоскости, чем вдоль оси $c$.

Мел к и й Al. Мелкие акцепторы Al обнаружены методами ОДМР [18-21] и обычным ЭПР $[11,16]$ в кристаллах $6 H$ - и $4 H$-SiC. ОДМР может наблюдаться благодаря наличию спиновых правил отбора для рекомбинации электронов и дырок (спин-зависимая донорно-акцепторная рекомбинация). Результаты измерений ОДМР по интенсивности донорно-акцепторной рекомбинации могут быть описаны спиновым гамильтонианом для слабо связанной донор-акцепторной пары:

$$
H=H_{\mathrm{D}}+H_{\mathrm{A}}+S_{\mathrm{D}} J S_{\mathrm{A}},
$$

где первый и второй члены описывают спиновые гамильтонианы для свободных доноров и акцепторов соответственно. Третий член описывает обменное взаимодействие между донором и акцептором. Для удаленных донорно-акцепторных пар это взаимодействие может быть гораздо меньше зеемановского взаимодействия доноров и акцепторов и, в результате, спектры ОДМР доноров и акцепторов могут быть уширены по сравнению со спектрами ЭПР изолированных примесей (например, наблюдаемых обычным методом ЭПР).

На рис. 5 показана часть спектра ОДМР мелких акцепторов $\mathrm{Al}$ при $1.5 \mathrm{~K}$ и угле $18^{\circ}$ между магнитным полем и осью $c$ кристалла. Сигнал ОДМР соответствует увеличению интенсивности люминесценции. Спектр состоит из сигнала $\mathrm{N}$ и группы из трех анизотропных линий. Эта группа может быть приписана мелким акцепторам Al в трех неэквивалентных положениях в решетке. На рис. $1, b$ показана угловая зависимость спектров ОДМР мелких акцепторов $\mathrm{Al}$ при вращении кристалла от В $\| c$ к $B \perp c$. СТ структура не наблюдалась. Спектры ОДМР могут быть описаны спиновым гамильтонианом с параметрами $S=1 / 2$ и $g_{\|}=2.412,2.400$ и $2.325 ; g_{\perp} \cong 0$; $g$-фактор подчиняется закону $g=g_{\|} \cos \theta$, где $\theta-$ угол между магнитным полем и осью $c$ кристалла. Такое поведение характерно для акцепторов, описываемых приближением эффективной массы, но с уменьшенным орбитальным моментом из-за локализации.

Мелкий $\mathrm{Ga}$. В кристаллах $6 H$-SiC, легированных галлием, был обнаружен сильно анизотропный сигнал магнитного резонанса мелких акцепторов $\mathrm{Ga}$ [19-21]. Спектр ОДМР мелкого Ga также показан на рис. 5. Сигнал соответствует увеличению интенсивности люминесценции. В отличие от мелкого $\mathrm{Al}$, мелкий $\mathrm{Ga}$ имеет разрешенную СТ структуру. Галлий имеет два стабильных изотопа ${ }^{69} \mathrm{Ga}$ и ${ }^{71} \mathrm{Ga}$ с природными распространенностями $60.1 \%$ и $39.9 \%$ соответственно. Оба 


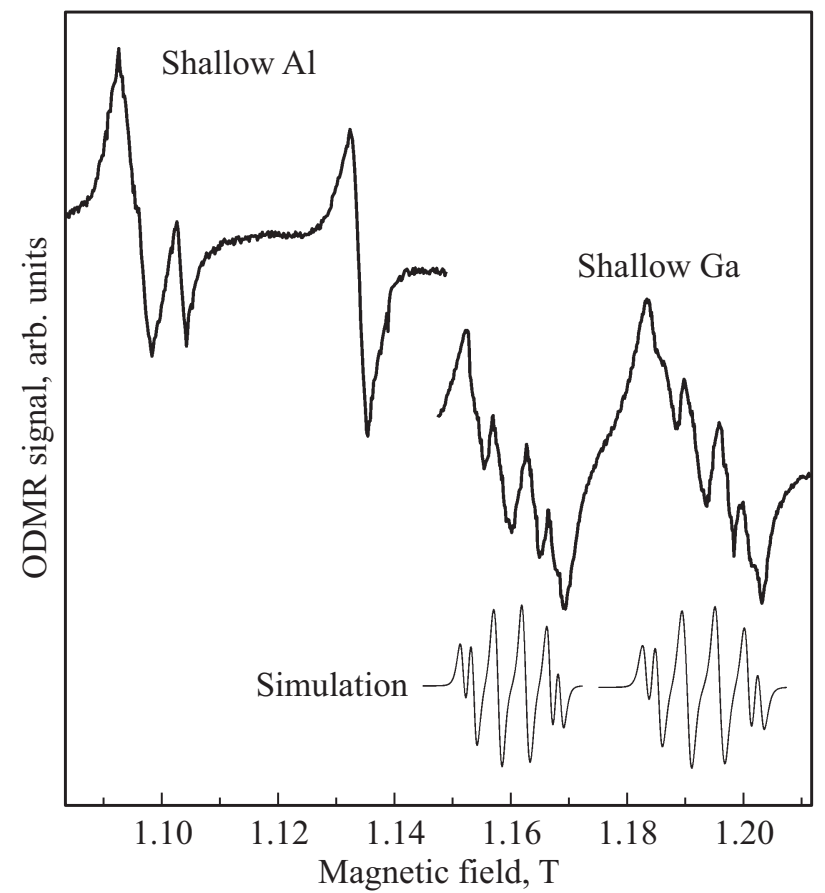

Рис. 5. Спектры ОДМР, зарегистрированные при $1.5 \mathrm{~K}$ и $35.2 \mathrm{GHz}$ в $6 H-\mathrm{SiC}: \mathrm{Ga}$ и $6 H-\mathrm{SiC}: \mathrm{Al}$, магнитное поле лежит в плоскости $\{11 \overline{2} 0\}$ и направлено под углом $18^{\circ}$ к оси $c$.

изотопа имеют ядерный спин $I=3 / 2$. Отношение ядерных $g$-факторов $g_{\mathrm{I}}\left({ }^{71} \mathrm{Ga}\right) / g_{\mathrm{I}}\left({ }^{69} \mathrm{Ga}\right)=1.27$, где $g_{\mathrm{I}}\left({ }^{69} \mathrm{Ga}\right)$ и $g_{\mathrm{I}}\left({ }^{71} \mathrm{Ga}\right)$ - ядерные $g$-факторы изотопов ${ }^{69} \mathrm{Ga}$ и ${ }^{71} \mathrm{Ga}$ соответственно. Так как ${ }^{69} \mathrm{Ga}$ имеет распространенность порядка $60 \%$, то ожидается спектр в виде одного или нескольких наборов из четырех линий одинаковой интенсивности. Изотоп ${ }^{71} \mathrm{Ga}$ приведет к появлению четырех более слабых линий с расстоянием в 1.27 раз больше (в соответствии с отношением ядерных $g$-факторов ${ }^{71} \mathrm{Ga}$ и ${ }^{69} \mathrm{Ga}$ ) по сравнению с линиями ${ }^{69} \mathrm{Ga}$.

Разрешенная СТ структура спектров ОДМР полностью соответствовала ожидаемой от взаимодействия неспаренного электрона с ядрами Ga. Для демонстрации этого соответствия был проведен расчет СТ структуры Ga. На рис. 5 показаны рассчитанная (внизу) СТ структура $\mathrm{Ga}$, которая прекрасно демонстрирует, что анизотропный сигнал ОДМР принадлежит акцепторам $\mathrm{Ga}$. Два сигнала $\mathrm{Ga}$ принадлежат примеси в разных позициях в решетке $(h, k 1, k 2)$. Две позиции (по-видимому, $k 1, k 2)$ не разрешаются.

Угловые зависимости ОДМР в $6 \mathrm{H}-\mathrm{SiC}: \mathrm{Ga}$ показаны на рис. $1, b$. Спектр может быть описан спиновым гамильтонианом с $S=1 / 2$ и $I=3 / 2$. Параметры сигналов ОДМР мелких акцепторов галлия следующие: $g_{\|}=$ $=2.27$ и 2.21. Для обоих сигналов $g_{\perp} \cong 0.6$. Константы СТ взаимодействия: 1) $A_{\|}\left({ }^{69} \mathrm{Ga}\right) \cong 34 \cdot 10^{-4} \mathrm{~cm}^{-1}$ и $A_{\|}\left({ }^{71} \mathrm{Ga}\right) \cong 43 \cdot 10^{-4} \mathrm{~cm}^{-1}$ для сигнала с $g_{\|}=2.27$; 2) $A_{\|}\left({ }^{69} \mathrm{Ga}\right) \cong 40 \cdot 10^{-4} \mathrm{~cm}^{-1}$ и $A_{\|}\left({ }^{71} \mathrm{Ga}\right) \cong 50 \cdot 10^{-4} \mathrm{~cm}^{-1}$ для сигнала с $g_{\|}=2.21$. Анизотропная часть СТ вза- имодействия, судя по всему, мала и не может быть определена из измерений ОДМР, таким образом, $A_{\|} \cong a$, где $a-$ изотропная часть СТ взаимодействия. Уменьшение анизотропии сигналов мелких акцепторов $\mathrm{Ga}$ по сравнению c Al может объясняться большей глубиной залегания уровней $\mathrm{Ga}$ (рис. $1, a$ ). Поведение мелких акцепторов $\mathrm{Ga}$ соответствует теории эффективной массы, но с уменьшенным вкладом орбитального момента из-за локализации, которая сильнее, чем в случае $\mathrm{Al}$.

Из величины CT взаимодействий с Ga может быть извлечена важная информация о степени локализации волновой функции неспаренного электрона. К сожалению, оказалось невозможным определить анизотропию СТ структуры, чтобы найти анизотропный член $b$. Мы можем только оценить анизотропную часть константы СТ взаимодействия. Можно предположить, что имеет место $s p^{3}$-гибридизация, и в этом случае спиновая плотность на орбитали $\mathrm{Ga}$ оценивается в $5 \%$ для сигнала с $g_{\|}=2.27$ и $6 \%$ для сигнала с $g_{\|}=2.21$. В таком случае анизотропный член $b \cong 0.05 \mathrm{mT}$ и эта величина лежит в пределах экспериментальной ошибки измерения СТ констант. Большая часть спиновой плотности, по-видимому, распределена по узлам, соседним с атомом Ga. Сигнал с $g_{\|}=2.27$, вероятно, соответствует акцептору Ga c более мелким уровнем, чем в случае $\mathrm{Ga}$ с $g_{\|}=2.21$. Таким образом, как следует из схемы энергетических уровней (рис. $1, a$ ), сигнал с $g_{\|}=2.27$ может быть приписан $\mathrm{Ga}$ в $h$-позиции, а сигнал с $g_{\|}=2.21-$ мелкому акцептору галлия в квазикубических позициях.

Используя результаты для мелких акцепторов $\mathrm{Ga}$, мы можем оценить из ширины линии ЭПР константы СТ взаимодействия для акцепторов $\mathrm{Al}$, которое не разрешается в спектрах ЭПР. В случае высоко- и низко-полевых линий СТ взаимодействие оценивается в $\sim 0.6 \mathrm{mT}$ и $\sim 0.7 \mathrm{mT}$ соответственно. Соответствующие спиновые плотности на орбитали $\mathrm{Al}$ оцениваются $\sim 2.5 \%$ для сигнала с $g_{\|}=2.412$ и $\sim 3 \%$ для сигнала с $g_{\|}=2.325$. Предполагается, что сигнал с $g_{\|}=2.412$ соответствует более мелкому акцептору $\mathrm{Al}$, чем $\mathrm{Al} \mathrm{c} g_{\|}=2.325$. Как показано на схеме энергетических уровней (рис. $1, a$ ), сигнал с $g_{\|}=2.412$ может быть приписан $h$-позиции акцептора $\mathrm{Al}$, а сигнал с $g_{\|}=2.325$ - алюминию в квазикубических позициях.

2.2. Акцепторы с глубокими уровнями.

В этом разделе будут представлены результаты исследований ЭПР, ДЭЯР и ОДМР глубоких акцепторов В, $\mathrm{Al}, \mathrm{Ga}$ в $\mathrm{SiC}$.

Гл убоки й $\mathrm{B}$. Первое наблюдение магнитного резонанса глубоких акцепторов В (deep B $-\mathrm{dB}$ ) в $\mathrm{SiC}$ было проведено с помощью техники ОДМР [20,21,32,33]. При $1.5 \mathrm{~K}$ в $6 H$ - и $4 H-\mathrm{SiC}$ наблюдалось несколько анизотропных сигналов ОДМР, приписанных глубоким акцепторам бора. ОДМР регистрировался по интенсивности желтой полосы люминесценции в $6 H$-SiC и ее аналогу в $4 H$-SiC. Анизотропные сигналы ОДМР принадлежат разным типам центров, так как их относительные интенсивности изменялись с изменением длины волны 



Рис. 6. (a) Угловая зависимость спектров ЭПР в $X$-диапазоне в кристалле $6 H$-SiC, легированном изотопом ${ }^{11} \mathrm{~B}$. Штриховые линии показывают спектры в кристалле $6 H-\mathrm{SiC}$, легированном изотопом ${ }^{10} \mathrm{~B}$. (b) Угловая зависимость спектров ЭПР в $X$-диапазоне в кристалле $6 H-\mathrm{SiC}: \mathrm{Ga}$. Рассчитанная зависимость для мелкого галлия показана вертикальными линиями. На вставке показаны экспериментальный сигнал с корректированной базовой линией (наверху) и результат расчета (внизу) для сверхтонкой структуры $\mathrm{Ga}$ при ориентации магнитного поля под углом $25^{\circ}$ относительно оси $c$.

излучения в пределах полосы люминесценции. Сигналы ОДМР $\mathrm{dB}$ имели почти аксиальную симметрию вокруг оси $c$ кристалла, $g_{\|} \cong 2.02-2.03$ и $g_{\perp} \cong 2.0$. Низкое разрешение метода ОДМР не позволило наблюдать СТ структуру спектров и однозначно приписать спектры примесям бора.

Результаты исследований ЭПР глубоких центров В представлены в работах [13-17,24]. Измерения ДЭЯР проводились в работах $[24,34,35]$. Спектры ЭПР, зарегистрированные в $X$-диапазоне в кристалле $6 H$ - $\mathrm{SiC}:{ }^{11} \mathrm{~B}$ при $4.5 \mathrm{~K}$ и различных ориентациях кристалла показаны на рис. $6, a$. Высокопольная часть спектров (в ориентациях, близких к $\mathbf{B} \| c$ ) в диапазоне 326.5-328.5 mT и низкопольная часть в диапазоне 323.0-326.0 $\mathrm{mT}$ принадлежат разным центрам, так как соотношение их интенсивностей различается для разных образцов. Высокопольный сигнал, обозначенный $\mathrm{SB}$ и отмеченный горизонтальной линией, принадлежит мелкому бору, так как этот сигнал может быть описан спиновым гамильтонианом с $g$-факторами и параметрами СТ структуры мелкого бора в двух квазикубических и гексагональном положениях в решетке. В области низких полей при углах, близких к $\theta=0^{\circ}$, наблюдаются две перекрывающиеся широкие неразрешенные линии, обозначенные на рис. $6, a$ как $\mathrm{dB}$. Интенсивность этих линий коррелирует с характерной светло-желтой люминесценцией и сигналами ОДМР глубоких центров бора. Высокотемпературный отжиг сильно влияет на низкополевой сигнал ЭПР. Пятиминутный отжиг при $2350^{\circ} \mathrm{C}$ приводит к резкому уменьшению указанного сигнала ЭПР и одновременному уменьшению интенсивности желтой люминесценции и сигналов DLTS глубоких центров бора. После отжига в образцах наблюдаются только сигналы мелких акцепторов бора. Слабый сигнал при $328.5 \mathrm{mT}$ принадлежит высокопольной компоненте СТ структуры азота.

Неразрешенные широкие линии на рис. 6,a были интерпретированы как возникающие из-за наличия в кристаллах глубоких центров бора в неэквивалентных положениях в решетке. Этот спектр анизотропен и при вращении магнитного поля от оси $c$ при углах, 
больших $20^{\circ}$, появляется разрешенная структура, и разрешение спектра улучшается при увеличении угла. Структура имеет наилучшее разрешение при $\theta \cong 40^{\circ}$. При $\theta>60^{\circ}$ разрешенная структура исчезает, и интенсивность сигнала ЭПР глубокого бора падает. Прямая идентификация примесей, входящих в состав дефекта, может быть получена наблюдением СТ структуры спектра. Чтобы однозначно продемонстрировать, что наблюдаемая в спектрах СТ структура обусловлена взаимодействием с ядрами ${ }^{11} \mathrm{~B}$, были проведены измерения ЭПР на кристаллах $\mathrm{SiC}$, легированных изотопом ${ }^{10} \mathrm{~B}$. Эти измерения однозначно показали, что разрешенная структура на рис. $6, a$ возникает из-за СТ взаимодействия спина неспаренного электрона с ядром изотопа ${ }^{11} \mathrm{~B}$ и, соответственно, сигнал обусловлен примесью бора. Идентификация бора, входящего в состав центра, была также проведена с помощью метода ДЭЯР [24,25,34].

В спектрах ЭПР глубоких центров бора $g$-тензор почти аксиален относительно оси $c$. Положения групп линий (которые принадлежат дефекту в неэквивалентных положениях в решетке), как и структура каждой группы, могут быть описаны аксиальным спиновым гамильтонианом, в котором ось $z$ совпадает с осью $c$ кристалла $S=1 / 2, I=3 / 2\left({ }^{11} \mathrm{~B}\right)$.

Значение $g_{\|}$оказалось зависимым от температуры. При нагревании кристалла выше 5 K эта линия смещается в область более высоких магнитных полей ( $g$-фактор уменьшается) и интенсивность ее падает, начиная с $10 \mathrm{~K}$. Разрешение спектров для ${ }^{11} \mathrm{~B}$, которое наблюдается при углах $\theta$ между $20^{\circ}$ и $60^{\circ}$, зависит от температуры, и при повышении температуры выше $8 \mathrm{~K}$ сигнал ЭПР уширяется и разрешенная СТ структура исчезает. Это уширение объясняется, возможно, термически активируемым движением дырок, связанных с глубоким бором.

Отметим, что на дефект, расположенный в $h$-узле, должно действовать более сильное локальное аксиальное поле, чем на дефект в $k$-узле. Разумно предположить, что из глубоких центров бора наибольший сдвиг $g$-фактора в случае магнитного поля, параллельного оси $c$ кристалла, имеет глубокий центр $\mathrm{B}$, занимающий $h$-узел. Более интенсивная линия с большей шириной на рис. 6, a (примерно в два раза более интенсивная), сдвинутая в область высоких магнитных полей, предположительно принадлежит двум неразрешенным квазикубическим позициям глубокого бора $k 1$ и $k 2$. При $T=4.5 \mathrm{~K} g_{\|} \cong 2.03$ и $g_{\perp} \cong 2.01$ для $h$-узла; $g_{\|} \cong 2.02$, 2.023 и $g_{\perp} \cong 2.0,1.99$ для квазикубических узлов. Изотропная часть СТ взаимодействия с ${ }^{11} \mathrm{~B}$ оценивается величиной $\sim 0.2 \mathrm{mT}$ для $h$-узла и $\sim 0.3 \mathrm{mT}$ для $k$-узлов. Анизотропная часть мала и не может быть определена из спектров ЭПР. Результаты измерений ДЭЯР глубокого бора в $X$-диапазоне [34] дают константы СТ структуры ${ }^{11} \mathrm{~B}: a=5.8 \mathrm{MHz}, b<0.3 \mathrm{MHz}(h$-узел); $a=6.1 \mathrm{MHz}, b \cong 1.5 \mathrm{MHz}$ и $a=6.2 \mathrm{MHz}, b \cong 1.5 \mathrm{MHz}$ (квазикубические узлы). Константа квадрупольного взаимодействия $Q$ для ядра ${ }^{11} \mathrm{~B}$ составляет $0.2 \mathrm{MHz}$ для $h$-узла и $0.19 \mathrm{MHz}$ для $k$-узлов бора. Следует отметить, что для глубокого бора константа $Q$ примерно в четыре раза меньше, чем для мелких акцепторов бора $(\sim 0.75 \mathrm{MHz})[26]$. Более того, константа СТ взаимодействия для мелкого бора резко анизотропна, в противоположность случаю глубокого бора. Анизотропная часть константы СТ структуры для глубокого бора почти полностью подавлена для $h$-позиций и частично подавлена для $k$-позиций. Спектры ЭПР глубокого бора также наблюдались в кристаллах $4 H$ - и $3 C-\mathrm{SiC}$ [31]. Предварительные результаты исследований глубокого бора в кубическом $\mathrm{SiC}$ показывают, что эти центры имеют ось симметрии $\langle 111\rangle$.

В кристаллах $6 H$-SiC с естественным содержанием изотопов, а также обогащенных (30\%) изотопом ${ }^{13} \mathrm{C}$ были проведены исследования ДЭЯР в $W$-диапазоне $(95 \mathrm{GHz})$. На рис. $3, b$ показаны спектры обоих образцов в ориентации $\mathbf{B} \| c$, на рисунке видны переходы ДЭЯР ядер ${ }^{29} \mathrm{Si} \quad(I=1 / 2),{ }^{13} \mathrm{C} \quad(I=1 / 2)$ и ${ }^{11} \mathrm{~B} \quad(I=3 / 2)$. Спектр вокруг ядерной зеемановской частоты кремния показан на вставке в увеличенном масштабе. Спектры ДЭЯР для мелкого $(a)$ и глубокого $(b)$ бора специально представлены на одном рисунке (рис. 3), чтобы можно было провести их сравнительный анализ.

Спектр ДЭЯР ${ }^{11} \mathrm{~B}$ на рис. $3, b$ состоит из трех узких линий и широкой неразрешенной линии, симметрично расположенных вокруг ядерной зеемановской частоты ${ }^{11} \mathrm{~B}$. Так как изотоп ${ }^{11} \mathrm{~B}$ имеет ядерный спин $I=3 / 2$, ожидается по три линии для каждой позиции с обеих сторон от ларморовской частоты ${ }^{11} \mathrm{~B}$ из-за квадрупольного взаимодействия. Значения квадрупольного и СТ взаимодействий ${ }^{11} \mathrm{~B}$, полученные из спектров ДЭЯР: $A_{\|}(k 1)=8.8 \mathrm{MHz}, A_{\|}(h)=5.4 \mathrm{MHz}$ и $P(k 1, h)=190 \mathrm{kHz}$, что соответствует данным ДЭЯР в $X$-диапазоне в работе [34].

Спектр ДЭЯР ${ }^{13} \mathrm{C}$ в ориентации В $\| c$ состоит из широкой линии вокруг ядерной зеемановской частоты ${ }^{13} \mathrm{C}$ и двух отдельных линий при $32.2 \mathrm{MHz}$ и $40.0 \mathrm{MHz}$, симметрично расположенных вокруг зеемановской частоты, которые соответствуют, вероятно, атомам ${ }^{13} \mathrm{C}$ с наивысшей спиновой плотностью. Константа СТ структуры $A_{\|}=7.8 \mathrm{MHz}$. В широкой линии в окрестности зеемановской частоты ${ }^{13} \mathrm{C}$ можно различить несколько линий с максимальным значением константы СТ взаимодействия $A_{\|}=2.2 \mathrm{MHz}$. Спектр ДЭЯР ${ }^{29} \mathrm{Si}$ состоит из широкой линии на зеемановской частоте ${ }^{29} \mathrm{Si}$. Можно различить две линии с СТ расщеплением $A_{\|}=0.76 \mathrm{MHz}$.

Глубокий Ga. Спектр ЭПР предположительно глубокого галлия наблюдался в эпитаксиальных слоях $6 H$-SiC, легированного $\mathrm{Ga}[16,17]$. Спектр, сильно зависящий от угла между осью $c$ кристалла и магнитным полем, показан в разных ориентациях магнитного поля на рис. $6, b$. Следует отметить большое качественное сходство между спектрами ЭПР глубоких центров бора (рис. $6, a$ ) и спектрами, наблюдаемыми в $6 \mathrm{H}-\mathrm{SiC}$, легированном Ga (рис. $6, b)$. Наблюдались только количествен- 
ные различия в параметрах спинового гамильтониана сигналов в кристаллах, легированных $\mathrm{Ga}$ и В. Как ясно видно на рис. $6, a$ и $6, b$, только сдвиг $g$-фактора и СТ расщепления отличаются для двух примесей. Спектр, приписанный центрам $\mathrm{Ga}$, анизотропен и при отклонении ориентации кристалла от оси $c$ разрешение спектра улучшается при увеличении угла. Структура имеет наилучшее разрешение при $\theta \sim 25^{\circ}$. Кроме того, сигнал с $g$-фактором $g \sim 2.0$ возникает из-за наличия в кристалле мелких акцепторов бора (В является неконтролируемой примесью в карбиде кремния). Наличие одного атома $\mathrm{Ga}$ в составе дефекта предполагается из-за наличия разрешенной СТ структуры. Для демонстрации того, что разрешенная СТ структура при углах, больших $20^{\circ}$, возникает из-за СТ взаимодействия с ядрами ${ }^{69} \mathrm{Ga}$ и ${ }^{71} \mathrm{Ga}$, был проведен расчет СТ структуры Ga. Вставка на рис. $6, b$ показывает экспериментальную (сверху) и рассчитанную (снизу) CT структуру Ga для магнитного поля, направленного под углом $25^{\circ}$ к оси $c$. Расчет основывался на предположении, что экспериментальный спектр может быть разложен на три набора СТ сигналов от изотопов ${ }^{69} \mathrm{Ga}$ и ${ }^{71} \mathrm{Ga}$, которые возникают из-за трех неэквивалентных положений $\mathrm{Ga}$ в решетке кристалла. Используя этот подход и слегка различные $g$-факторы (слегка сдвинутые центры тяжести СТ структур) центров $\mathrm{Ga}$ для трех положений в решетке $\left(g_{1}=2.121\right.$, $g_{2}=2.096$ и $\left.g_{3}=2.070\right)$, оказалось возможным удачно воспроизвести экспериментальный спектр. Как и ожидалось, получено разумное соответствие между экспериментом и расчетом при использовании отношения между СТ параметрами для двух изотопов, соответствующего отношению ядерных $g$-факторов изотопов галлия. Была использована одна константа СТ взаимодействия $\left(4 \mathrm{mT}\right.$, относящаяся к $\left.{ }^{69} \mathrm{Ga}\right)$ для всех трех положений примеси в решетке, что, вероятно, является грубым приближением. Этот анализ СТ взаимодействия с $\mathrm{Ga}$ однозначно показал, что в состав обнаруженного дефекта входит один атом Ga. Таким образом, можно

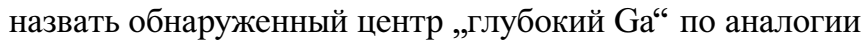
с глубоким бором. Отношение констант СТ структуры глубоких $\mathrm{Ga}$ и В соответствует отношению теоретических констант СТ взаимодействия для атомов $\mathrm{Ga}$ и B [36].

Тензор $g$-факторов спектров ЭПР глубоких центров $\mathrm{Ga}$, как и для глубокого бора, почти аксиален относительно оси $c$, и положения групп линий могут быть описаны тем же стандартным спиновым гамильтонианом. Главные значения $g$-тензора при $T=4.5 \mathrm{~K}$ составляют $g_{\|} \cong 2.16-2.19$ и $g_{\perp} \cong 2.0$, то есть для глубокого $\mathrm{Ga}$ не наблюдается поведения, описываемого в рамках теории эффективной массы. Интересно сравнить физическое поведение хорошо известных мелких акцепторов $\mathrm{Ga}$ [19-21] и новых центров Ga. Рассчитанные угловые зависимости ЭПР-переходов для мелких акцепторов $\mathrm{Ga}$ по данным ОДМР [19] (СТ структура не показана, то есть положения линий указаны как середины СТ структур) показаны линиями. Разница в положениях линий для мелких и глубоких акцепторов $\mathrm{Ga}$ очевидна при углах, превышающих $30^{\circ}$. Видно, что при ориентациях, близких к $\theta=0^{\circ}$, спектры ЭПР мелких и глубоких акцепторов $\mathrm{Ga}$ перекрываются, однако в наших экспериментах интенсивность сигналов ЭПР мелкого Ga слишком мала и не препятствует наблюдению сигналов глубоких акцепторов $\mathrm{Ga}$.

Как и в случае глубоких центров бора, величина $g_{\|}$ для глубокого $\mathrm{Ga}$ зависит от температуры. При нагревании кристалла $\left(\theta=0^{\circ}\right)$ выше $5 \mathrm{~K}$ линии сдвигаются в область более высоких магнитных полей ( $g$-фактор уменьшается) и их интенсивность снижается, начиная с 8 K. Разрешение сигналов также зависит от температуры, и при повышении температуры до $6 \mathrm{~K}$ разрешенная СТ структура $\left(\theta=30^{\circ}\right)$ начинает исчезать.

Гл уб оки й A1. Обнаружение и установление структуры акцепторов алюминия с глубокими уровнями (глубокого алюминия) в карбиде кремния представляет большой интерес с точки зрения его применения, поскольку именно алюминий является основной акцепторной примесью в приборах на основе $\mathrm{SiC}$. При этом образование акцепторов алюминия с глубокими уровнями ухудшает электрические свойства материала, таким образом, важной проблемой является минимизация содержания глубокого алюминия в приборных структурах. Поэтому стояла задача обнаружить сигналы ЭПР в $\mathrm{SiC}$, легированном Al, у которых угловая и температурная зависимости похожи на те, что наблюдались для глубокого В и Ga. B работах [16,17] наблюдались сигналы ЭПР, которые были приписаны центрам глубокого алюминия в карбиде кремния. Важным аргументом в пользу возможности приписать эти сигналы ЭПР глубокому алюминию является наличие угловой и температурной зависимости сигналов ЭПР, подобных тем, что наблюдались для глубокого В и глубокого $\mathrm{Ga}$.

На рис. 7 показана угловая зависимость спектров ЭПР (X-диапазон), обнаруженных при $4.5 \mathrm{~K}$ в кристаллах $6 H$-SiC, легированных Al. Магнитное поле вращается в плоскости $\{11 \overline{2} 0\}$. Спектры состоят из нескольких групп линий, которые сильно зависят от угла между магнитным полем и осью $c$ кристалла. Одна из групп анизотропных линий принадлежит мелким акцепторам Al в неэквивалентных положениях в решетке. Эта группа видна в области низких магнитных полей на рис. 7 в виде двух широких линий (в ориентациях, близких к $\mathbf{B} \| c$ ). Угловая зависимость переходов ЭПР мелких акцепторов $\mathrm{Al}$ вида $\cos \theta$ показана вертикальными линиями. Такое поведение указывает на то, что мелкий акцептор ведет себя в рамках приближения эффективной массы. Некоторое несовпадение положения линий ЭПР и стрелок может объясняться неточной ориентацией образца. При нагревании кристалла сигналы ЭПР мелких акцепторов Al исчезают при температуре выше $5 \mathrm{~K}$. Другая группа анизотропных линий имеет угловую и температурную зависимость, похожую на те, что наблюдались для глубокого B или Ga. Поэтому мы будем обозначать новый центр Al как „глубокий $\mathrm{Al}^{“}$, аналогично глубокому B 


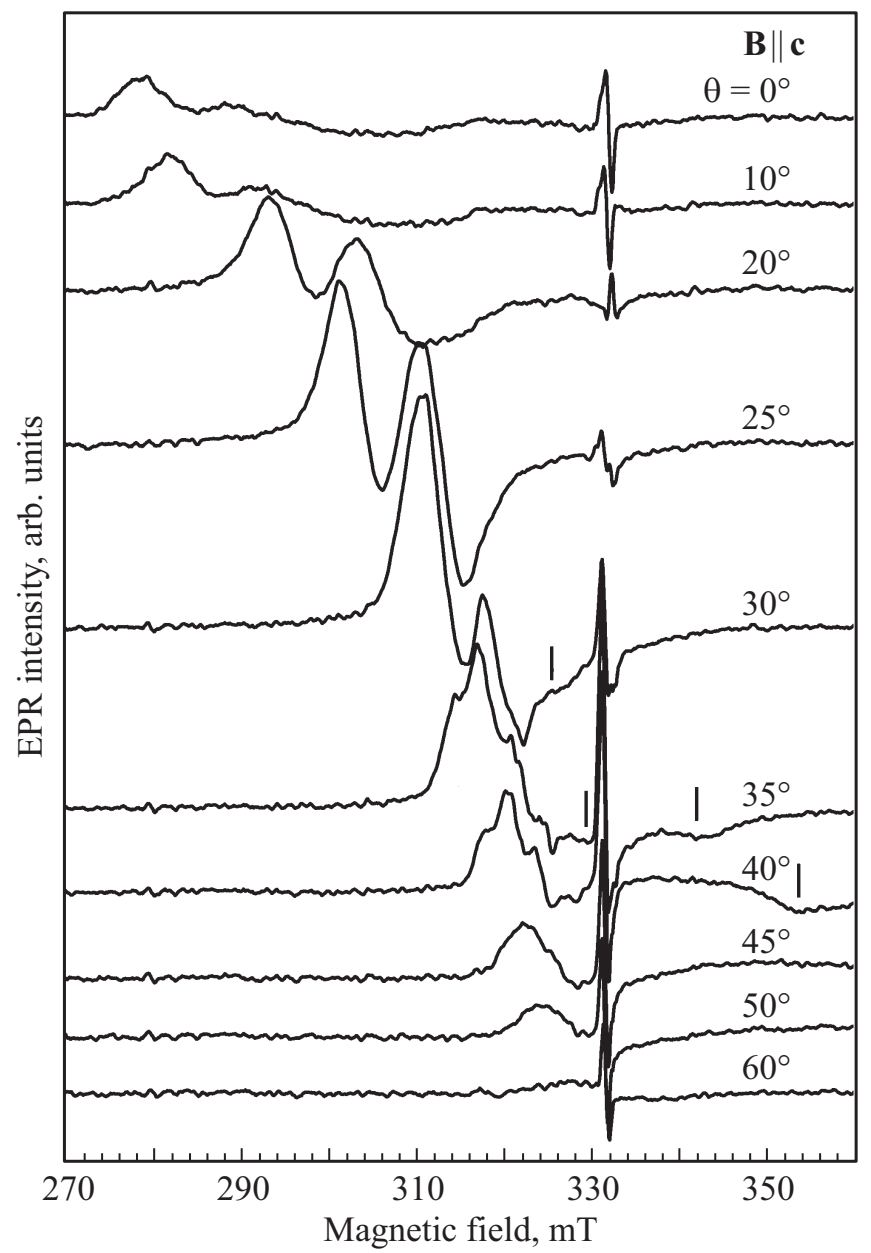

Рис. 7. Угловая зависимость спектров ЭПР в $X$-диапазона в кристалле $6 H$-SiC:Al p-типа. Теоретическая зависимость для мелкого алюминия показана вертикальными линиями.

и глубокому Ga. Различить сигналы мелких и глубоких акцепторов Al можно по температурной зависимости: сигналы глубокого Al имеют высокую интенсивность до $7 \mathrm{~K}$, в то время как сигналы мелкого Al исчезают при температуре выше $5 \mathrm{~K}$. Разница в положениях линий мелких и глубоких акцепторов Al отчетливо видна при углах, больших $30^{\circ}$, как и в случае Ga. При ориентациях, близких к $\theta=0^{\circ}$, сигналы мелкого и глубокого $\mathrm{Al}$ перекрываются.

У Al имеется один стабильный изотоп ${ }^{27} \mathrm{Al}$ с естественной распространенностью $100 \%$ и ядерным спином $I=5 / 2$. Таким образом, можно ожидать спектр в виде одного или нескольких наборов линий одинаковой интенсивности, расстояние между которыми примерно в четыре раза меньше (в соответствии с отношениями теоретически рассчитанных величин СТ констант атомов ${ }^{69} \mathrm{Ga}$ и $\left.{ }^{27} \mathrm{Al}[36]\right)$, чем у изотопа ${ }^{69} \mathrm{Ga}$.

Главные значения $g$-тензора при $T=4.5 \mathrm{~K}$ для глубокого $\mathrm{Al}$ в $6 H$-SiC оцениваются величинами $g_{\|} \cong 2.1-2.19$ и $g_{\perp} \cong 2.0$, то есть не наблюдается поведения, описываемого в приближении эффективной массы.
В диапазоне температур $\sim 4-10 \mathrm{~K}$ вид спектра ЭПР глубокого $\mathrm{Al}$ в $6 \mathrm{H}$-SiC резко меняется. При нагреве кристалла $\left(\theta=0^{\circ}\right)$ выше $5 \mathrm{~K}$ сигналы ЭПР мелких акцепторов Al исчезают, и сигнал глубокого Al сдвигается в область высоких магнитных полей и исчезает примерно при $10 \mathrm{~K}$. Таким образом, исходя из сходства температурных зависимостей спектров ЭПР, новый центр Al принадлежит к тому же типу центров, что глубокий В или глубокий $\mathrm{Ga}$.

Были проведены контрольные эксперименты на образцах $p$-типа $6 \mathrm{H}$-SiC, легированных $\mathrm{Al}$, выращенных Cree Corp. В этих образцах наблюдались интенсивные сигналы глубокого Al, концентрация которого сравнима с концентрацией мелких акцепторов Al. Таким образом, получено прямое свидетельство наличия глубоких уровней $\mathrm{Al}$ в пластинах $\mathrm{SiC} p$-типа, использующихся для применений в приборостроении.

Глубокие акцепторы $\mathrm{Al}$ в $4 \mathrm{H}-\mathrm{SiC}$ были исследованы и идентифицированы с использованием ЭПР и ДЭЯР в $X$-диапазоне [35]. На рис. 8, $a$ показаны спектры ЭПР, записанные при $4.5 \mathrm{~K}$ в нескольких ориентациях $4 H-\mathrm{SiC}$ $p$-типа, сильно легированного $\mathrm{Al}\left(N_{\mathrm{Al}}=10^{20} \mathrm{~cm}^{-3}\right)$. Легирование производилось в процессе роста, кристалл также содержит неконтролируемую примесь бора с концентрацией $10^{17} \mathrm{~cm}^{-3}$. В ориентации $\mathbf{B} \| c\left(\theta=0^{\circ}\right)$ видны три сигнала ЭПР. Один сигнал (состоящий из двух широких линий) обозначен $d \mathrm{Al}$, глубокий $\mathrm{Al}$. Угловая зависимость этого сигнала очень близка к той, что наблюдалась для глубокого $\mathrm{Al}$ в $6 \mathrm{H}$-SiC. Симметрия этого центра практически аксиальная относительно оси $c$ кристалла, сигнал ЭПР может быть описан стандартным спиновым гамильтонианом с электронным спином $S=1 / 2$ и $g$-факторами $g_{\|}=2.35 ; 2.23$. Две линии $d \mathrm{Al}$ присутствуют благодаря наличию примеси Al в двух неэквивалентных узлах в решетке $(h$ и $k)$. Как и в случае глубокого $\mathrm{Al}$ и глубокого В в $6 H-\mathrm{SiC}$, мы считаем, что низкополевой сигнал может быть приписан Al в $h$-позиции решетки. В отличие от предыдущих наблюдений глубокого $\mathrm{Al}$ в $6 H-\mathrm{SiC}$, в этом кристалле сигнал ЭПР глубокого алюминия не перекрывается сигналами от мелких акцепторов Al. Интенсивность сигналов ЭПР мелких акцепторов Al как минимум в 200 раз меньше, чем сигнала ЭПР глубокого Al.

Линия, обозначенная на рис. 8, $a$ как $\mathrm{sB}$, относится к мелким акцепторам В. Бор является обычной неконтролируемой примесью в кристаллах $\mathrm{SiC} p$-типа. Сигнал, отмеченный на рис. 8, $a$ как $\mathrm{dB}$ (глубокий В), имеет угловую и температурную зависимости, аналогичные сигналам глубокого бора, которые ранее наблюдались в кристаллах $4 \mathrm{H}-\mathrm{SiC}$, легированных в процессе диффузии. На вставке показан сигнал $d \mathrm{~B}$ в ориентации $\theta=40^{\circ}$, и видна характерная СТ структура от ядерного спина изотопа ${ }^{11} \mathrm{~B}(I=3 / 2)$ для двух позиций бора в решетке. Этот сигнал можно приписать глубоким акцепторам бора. Это весьма интересно, так как до этого глубокий бор наблюдался только в кристаллах, легированных в процессе диффузии, в то время как этот образец 

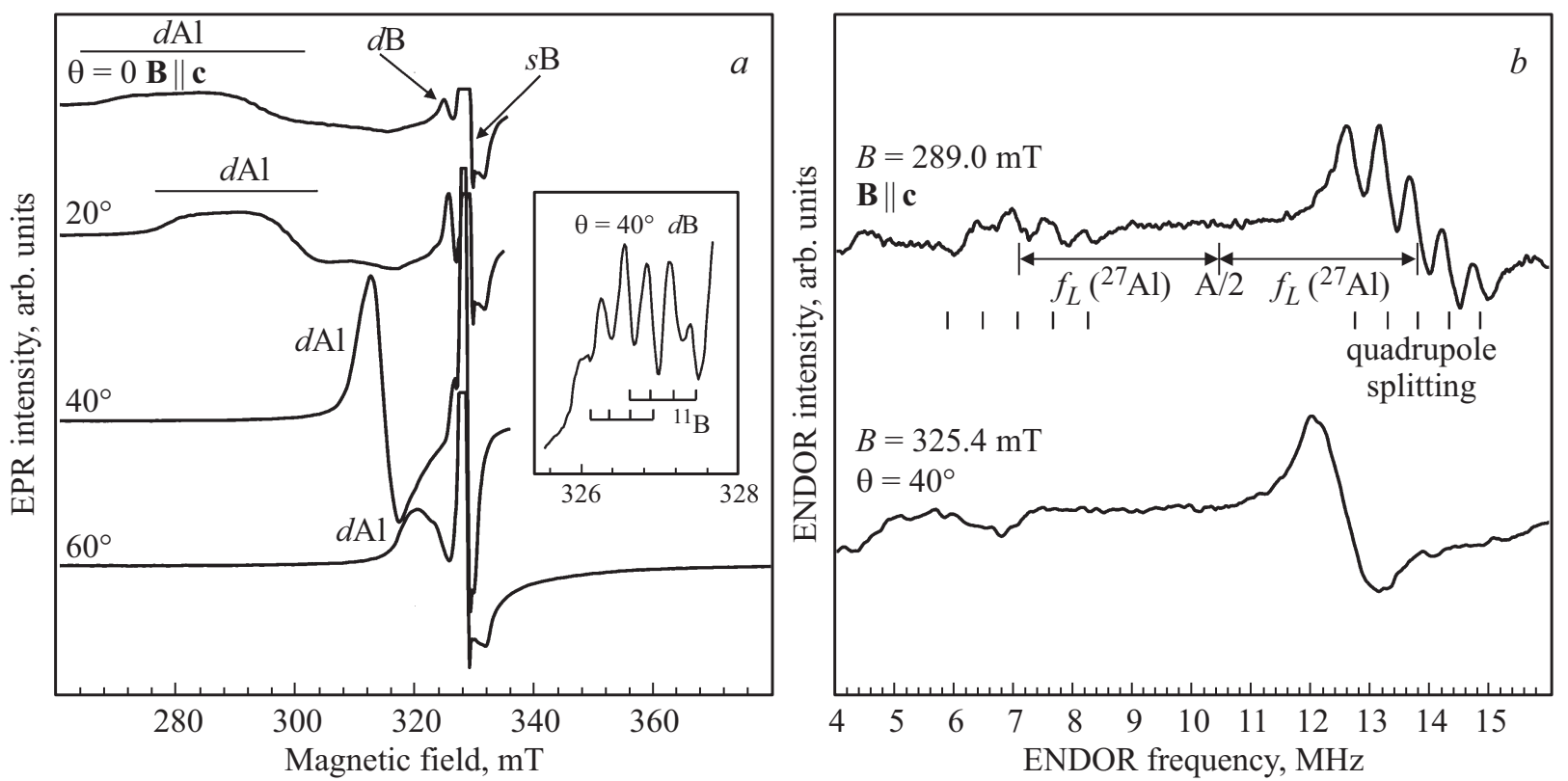

Рис. 8. (a) Спектры ЭПР, зарегистрированные при $4.5 \mathrm{~K}$ в различных ориентациях кристалла $4 H$-SiC, легированного $\mathrm{Al}$. Линия в центре может быть приписана бору, который имеет мелкие и глубокие акцепторные уровни и является неконтролируемой примесью в $\mathrm{SiC}$. На вставке показан сигнал ЭПР глубокого В при $\theta=40^{\circ}$. (b) Сигнал ДЭЯР глубокого акцептора Al, две группы линий отстоят на величину ларморовской частоты ядра ${ }^{27} \mathrm{Al}$ от положения, соответствующего половине величины СТ взаимодействия ${ }^{27} \mathrm{Al}$ (обозначено $A / 2$ ). Структура из пяти линий возникает из-за квадрупольного взаимодействия.
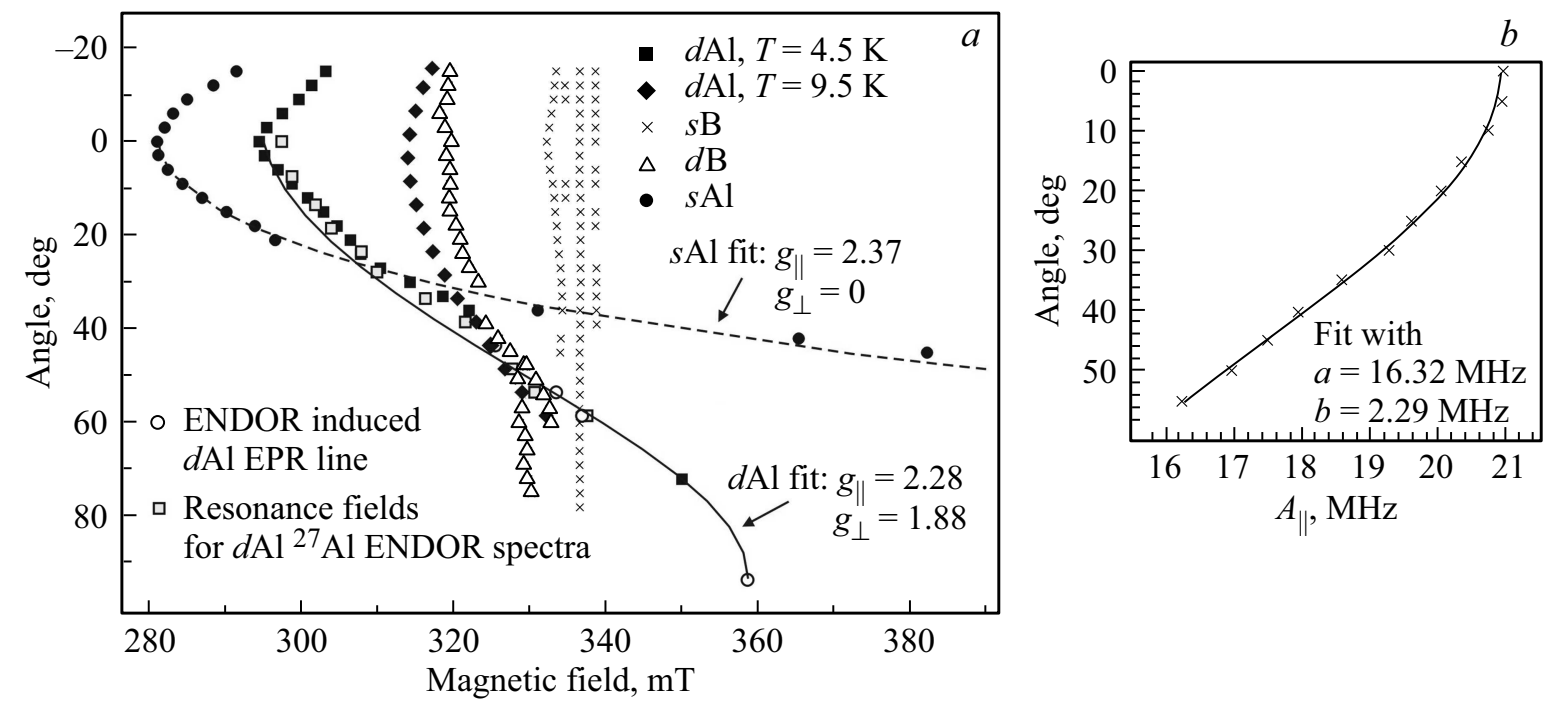

Рис. 9. (a) Угловая зависимость спектра ЭПР в $X$-диапазоне $(9.45 \mathrm{GHz})$ при $T=4.5 \mathrm{~K}$ в $4 H$-SiC: Аl; вращение осуществлялось в плоскости $\{11 \overline{2} 0\}$. (b)Угловая зависимость СТ расщепления $A_{\|}$, полученного по данным ДЭЯР, и расчет (сплошная линия) с учетом изотропной $(a)$ и анизотропной $(b)$ частей СТ взаимодействия.

легирован в процессе роста и бор представляет собой неконтролируемую примесь.

Для определения анизотропии $g$-фактора были проведены измерения угловой зависимости сигналов ЭПР. На рис. 9, $a$ показана угловая зависимость сигналов ЭПР в $X$-диапазоне в $4 H$-SiC: $\mathrm{Al}$ при $T=4.5 \mathrm{~K}$, вращение в плоскости $\{11 \overline{2} 0\}$. Кружками показаны положения мелких акцепторов $\mathrm{Al}$, квадратами - глубоких акцеп- торов Al. Видно, что при ориентации примерно $60^{\circ}$ по отношению к оси $c$ сигнал $\mathrm{Al}$ смешивается с сигналом мелкого бора, что ограничивает точность определения $g$-фактора в перпендикулярной ориентации (см. ниже).

Для лучшего понимания структуры центра были проведены измерения ДЭЯР. Ядерные магнитные резонансные переходы индуцировались между состояниями ядерных спинов атомов $\mathrm{Si}$ и $\mathrm{C}$, окружающих дефект 

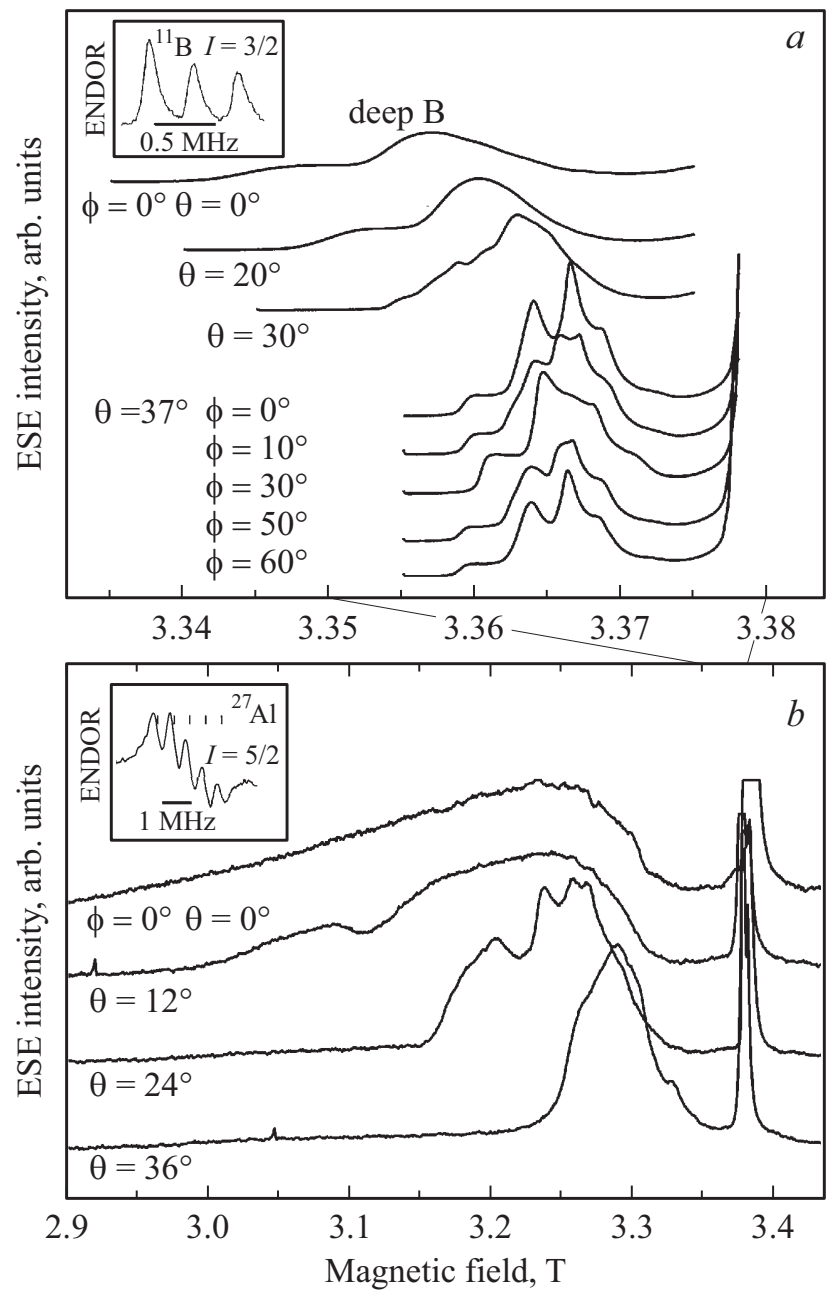

Рис. 10. Угловые зависимости спектров ЭПР глубокого бора, зарегистрированные на высокой частоте в $W$-диапазоне, $95 \mathrm{GHz}(a)$ и глубоких акцепторов $\mathrm{Al}(b)$ при $1.5 \mathrm{~K}$ в $6 H-\mathrm{SiC}$. Вращение в плоскости $\{11 \overline{2} 0\}, \theta-$ угол между направлением магнитного поля $\mathbf{B}$ и осью $c$. Для глубокого бора показана дополнительная зависимость от азимутального угла $\phi$ при постоянном угле $\theta=37^{\circ}$, то есть зависимость от $\phi$, с магнитным полем, описывающим конус при фиксированном угле $\theta$. Очевидна небольшая неаксиальность $g$-тензора. Вставки показывают спектры ДЭЯР, являющиеся характеристиками бора и алюминия.

и примесный атом, входящие в состав центра Идентификация основана на ядерном зеемановском взаимодействии, которое включает ларморовскую частоту соответствующего ядра. Для спина $S=1 / 2$ при наличии СТ взаимодействий в спектрах ДЭЯР наблюдаются пары линий (см. рис. $8, b)$, разделенные удвоенной ларморовской частотой ядра и расположенные вокруг частоты, составляющей примерно половину константы СТ взаимодействия. Характерный спектр, состоящий из пяти линий, определяется ядерным квадрупольным взаимодействием с одним ядром Al, имеющим ядерный спин $I=5 / 2$. Это, вместе с ларморовской частотой, однозначно указывает на $\mathrm{Al}$ как на примесь, входящую в состав дефекта. На рис. 9, $b$ показана угловая зависимость СТ расщепления $A$, полученная из данных ДЭЯР и расчет (сплошная линия), сделанный с использованием изотропной $(a)$ и анизотропной $(b)$ частей СТ взаимодействия.

Эта зависимость была измерена для различных ориентаций кристалла относительно магнитного поля (спектр ДЭЯР для $\theta=40^{\circ}$ также показан на рис. $\left.8, b\right)$ и позволила установить анизотропию $g$-фактора линии ЭПР. Высокочастотная линия на рис. $8, b$ может быть использована для измерения аналога спектра возбуждения ДЭЯР, так как она индуцируется только в пределах линии ЭПР дефекта. Это обычно называют ЭПР, индуцированный двойным электронно-ядерным резонансом (ENDOR tagged EPR). Так как ЭПР глубоких центров Al зависит от ориентации и, следовательно, наблюдается при различных магнитных полях, то необходимо соответственно подстраивать ядерную ларморовскую частоту. Светлые кружки на рис. 9 дают точные положения линии ЭПР алюминия, полученные с помощью ЭПР, индуцированного ДЭЯР (см. выше), описываемые $g$-факторами $g_{\|}=2.28$ и $g_{\perp}=1.88$ при $T=4.5 \mathrm{~K}$. Отметим, что для мелких акцепторов алюминия $g_{\|}=2.37$ и $g_{\perp}=0$.

Сигналы ЭПР глубокого Al демонстрируют сильную температурную зависимость. Анизотропия сигналов ЭПР уменьшается с увеличением температуры и при высоких температурах сигнал практически изотропен с $g=2.0$.

На рис. 10 показана угловая зависимость глубоких акцепторов бора $(a)$ и алюминия $(b)$ на частоте $95 \mathrm{GHz}$ при $1.5 \mathrm{~K}$ в $6 H$-SiC при вращении магнитного поля в плоскости $\{11 \overline{2} 0\}$. Углы $\theta$ и $\phi$ описывают ориентацию магнитного поля относительно оси $c$ и ориентацию магнитного поля в плоскости, перпендикулярной оси $c$, соответственно. Из рисунка видно, что главные оси $\mathrm{z}$ $g$-тензора для всех центров совпадают с осью $c$. Слабая неаксиальность $g$-тензора хорошо заметна. Вставки показывают спектры ДЭЯР, представляющие собой „отпечатки пальцев“ (,finger print") бора и алюминия и их квадрупольные расщепления.

\section{3. Обсуждение результатов}

Ме лки е акцеп т оры. Результаты для мелкого бора резко отличаются от тех, что получены для мелких акцепторов III группы, $\mathrm{Al}$ и $\mathrm{Ga}$ (рис. 1,b). В отличие от $\mathrm{Al}$ и $\mathrm{Ga}$, для мелких акцепторов бора (и акцепторов $\mathrm{Be}$ [37]) не наблюдается поведения, соответствующего теории эффективной массы. Основная проблема - понять, почему мелкие акцепторы $\mathrm{Al}$ и Ga демонстрируют поведение, описывающееся теорией эффективной массы, а мелкие акцепторы В (и Ве [37]) - нет. Одним из объяснений может быть положение энергетических уровней: лучшее соответствие $g$-факторам свободных дырок ожидается для акцепторов с наименьшей энергией связи, что экспериментально подтверждается для Al и $\mathrm{Ga}$. Однако это не так для мелкого В (и $\mathrm{Be})$, несмотря 
на то, что положения акцепторных уровней бора в запрещенной зоне находятся между уровнями мелкого $\mathrm{Al}$ и мелкого $\mathrm{Ga}$ (рис. 1,a).

Структура мелких акцепторов бора интерпретировалась несколько раз, и было предложено несколько противоречивых моделей. После первой публикации долгое время считалось, что В замещает С, так как наблюдалось только ССТ взаимодействие с ${ }^{29} \mathrm{Si}$. Результаты ЭПР-экспериментов на образцах $6 H$-SiC, обогащенных изотопом ${ }^{13} \mathrm{C}[10]$, и обнаруженное большое анизотропное ССТ взаимодействие с одним ядром ${ }^{13} \mathrm{C}$ показали, что В занимает узел $\mathrm{Si}$, а основная спиновая плотность локализована на оборванной связи соседнего атома С вдоль связи $\mathrm{C}-\mathrm{B}$. Наблюдаемое ССТ взаимодействие с ${ }^{29} \mathrm{Si}$ было приписано трем атомам кремния, соседствующим с C, несущим основную спиновую плотность.

Как было указано, удивительным свойством акцепторов В является практически отсутствующая анизотропия $g$-тензора (средний $g \cong 2$, рис. $1, b$ ), что резко отличает бор от мелких акцепторов $\mathrm{Al}$ и $\mathrm{Ga}$. Эта разница была объяснена в работах $[16,17]$ путем рассмотрения ионных радиусов примесей. Было предположено, что В, имеющий атомный радиус, меньший чем $\mathrm{Si}$, смещается от соседнего атома С вдоль направления $\mathrm{C}-\mathrm{B}$. В этой модели В имеет нейтральное зарядовое состояние. Похожее смещение, но в результате эффекта Яна-Теллера для В в $h$-узле $6 H$-SiC было предложено в работе [26], а в $3 C$-SiC - в работе [12]. Кластерные расчеты мелких центров бора в $3 C$-SiC с использованием самосогласованного метода Харти-Фока были проведены в работе [27]. Эти расчеты подтвердили нецентральное положение В в кубическом $\mathrm{SiC}$. В работе [28] была предложена альтернативная модель, в которой валентный электрон соседнего C переходит на атом В, таким образом формируя связь $\mathrm{B}-\mathrm{Si}-\mathrm{C}^{+}$, в которой В имеет отрицательный заряд. Неспаренный электрон $\mathrm{C}^{+}$равномерно распределен между тремя оставшимися связывающими орбиталями, В и С сдвигаются по направлению друг к другу.

Наличие двух альтернативных моделей мотивировало авторов работы [23] на измерения ЭПР и ДЭЯР в $W$-диапазоне $(95 \mathrm{GHz})$ в образце $6 \mathrm{H}-\mathrm{SiC}$, обогащенном изотопом ${ }^{13} \mathrm{C}$. Результаты этих экспериментов подтвердили модель мелкого В, предложенную в работах $[10,16,17,23,25,26]$, поэтому в настоящем обзоре обсуждается только эта модель.

Мелкий В замещает $\mathrm{Si}\left(\mathrm{B}_{\mathrm{Si}}\right)$, но до $40 \%$ неспаренной спиновой плотности локализовано на одном из ближайших атомов $\mathrm{C}$. $\mathrm{Al}$ и $\mathrm{Ga}$ с мелкими уровнями также замещают кремний в решетке $\mathrm{SiC}$. Разница между атомными радиусами замещающих акцепторов и Si $\Delta R=\left(R\left[A^{3+}\right]-R\left[\mathrm{Si}^{4+}\right]\right)$ составляет $-0.19,+0.09$ и $+0.20 \AA$ для $A=\mathrm{B}$, Al и $\mathrm{Ga}$ соответственно. Величины ионных радиусов рассчитывались в упрощенном приближении, в котором $\mathrm{SiC}$ состоит из ионов $\mathrm{Si}^{4+}$ и спаренных связывающих электронов. В такой модели акцептор имеет состояние $\mathrm{A}^{3+}$. Ионные радиусы $\mathrm{Si}^{4+}$ и $A^{3+}$, где $A=\mathrm{B}, \mathrm{Al}$ и Ga взяты из таблиц Брукера.
Физическое поведение мелких акцепторов в $\mathrm{SiC}$ зависит от размера акцептора: больше он (Al, $\mathrm{Ga})$ или меньше (B), чем Si. В полупроводниках существует механизм, который приводит к смещению некоторых примесей, например, кислорода в кремнии или азота в алмазе и кремнии из центрального замещающего положения. В работах $[38,39]$ авторы предположили, что за сдвиг примеси в нецентральное положение может отвечать сильное химическое пересвязывание. Допустим, мы поместили В в центр неискаженного тетраэдра гибридизованных углеродов. Общая энергия может быть записана как сумма связывающей энергии и энергии отталкивания. В случае атома В в узле $\mathrm{Si}$ совокупность сил приводит к выталкиванию примеси В в нецентральное положение. Нестабильность, приводящая к нецентральному положению, имеет природу химического пересвязывания. Для атома В оказывается энергетически более выгодным сместиться из центрального положения в узле для формирования сильных связей с тремя атомами углерода, чем оставаться в центральном положении со слабыми связями с четырьмя соседними атомами углерода. Если четыре атома углерода находятся на своих местах в идеальной решетке, то расстояние В-C составляет $\sim 1.89 \AA$, что в 1.21 раз больше, чем нормальное расстояние $\mathrm{C}-\mathrm{B}$ в $\mathrm{BC}_{3}$ (например, в молекуле $\mathrm{B}\left(\mathrm{CH}_{3}\right)_{3}[40]$ ), которое составляет $1.56 \AA$. Мы рассматриваем смещение атома В из центрального положения в узле $\mathrm{Si}$ вдоль направления связи $\mathrm{Si}-\mathrm{C}$ по направлению к центральной точке между трема атомами С, это положение энергетически наиболее выгодно. На рис. 11 представлены модели мелкого акцептора В, где показаны направления сдвига атома В из центрального положения и положение атома C, на котором локализована основная спиновая плотность в аксиальной $h$-позиции $(a)$ и неаксиальной $k$-позиции $(b)$. Локальные оси $z$ и $x$ лежат в плоскости $\{11 \overline{2} 0\}$, указанной на рисунке, будучи главными осями $g$-тензора и $A$-тензора.

В рамках этой модели важный пункт - изменение в гибридизации атома В. Связи между атомом В и четырьмя соседними атомами С могут отличаться от обычных $s p^{3}$-гибридованных орбиталей. Если атом В смещается к центральной точке между тремя атомами углерода, то молекула $\mathrm{BC}_{3}$, состоящая из атома В и трех атомов углерода, не имеет обычной $s p^{3}$-гибридизации и релаксирует к $s p^{2}$-гибридизации. Гибридные орбитали $s p^{2}$ (естественные для В) направлены к атому В в планарной конфигурации. В таком случае нестабильность приводит к сдвигу атома В или в направлении $c$ (в случае $h$-позиции В) или в одном из шести направлений $\mathrm{Si}-\mathrm{C}$, не совпадающих с осью $c$ (в случае $k 1-$ и $k 2$-позиции В в $6 H-\mathrm{SiC})$. В кубическом $3 C-\mathrm{SiC}$ В сдвигается в одном из направлений $\langle 111\rangle$. Примерно 40\% спиновой плотности локализовано на $p_{z}$-орбитали атома $\mathrm{C}$, который сдвигается в направлении, противоположном атому В. Эта модель позволяет получить качественное объяснение свойств $g$-тензора. Предположим, что неспаренный электрон занимает $p_{z}$-орбиталь, направленную 


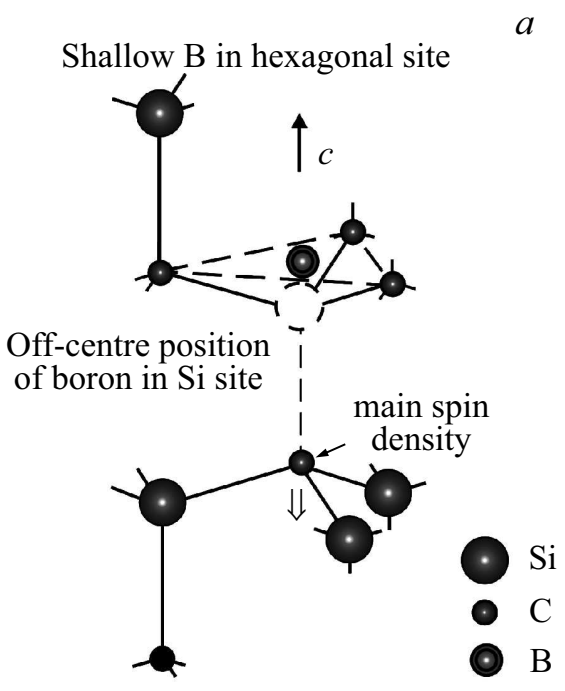

Shallow B in cubic-like site

$b$


Рис. 11. Модель мелкого В для гексагональной $(a)$ и квазикубической $(b)$ позиций в $\mathrm{SiC}$. Атом В сдвинут из центральной позиции вдоль локальной оси $z$. Двойная стрелка показывает сдвиг атома С, несущего основную спиновую плотность. (c) Модель глубокого акцептора бора. Распределение спиновой плотности глубокого бора (гексагональная позиция). В $(\mathrm{Si})$ - положение примеси бора в узле Si. $V(\mathrm{C})$ - положение вакансии углерода. (d) Простая одноэлектронная модель LCAO-MO для электронной структуры глубокого акцептора бора. Связь В-V направлена вдоль оси $c$ (направление [111] в кубе). $a, b$, и $c$ - три оборванных связи атомов $\mathrm{Si}$, окружающих вакансию. Без учета ян-теллеровского искажения точечная группа симметрии $C_{3 v}$, с учетом эффекта Яна-Теллера симметрия $C_{1 h}$.

к атому В. В таком случае $g$-тензор и $A$-тензор имеют цилиндрическую симметрию относительно оси $p_{z}$-орбитали, которая совпадает с осью $c$ для аксиального центра $\left(h\right.$-узел) или составляет угол $110^{\circ}\left(70^{\circ}\right)$ с осью $c$ для неаксиальных центров (позиции $k 1$ или $k 2$ ). Главные значения $g$-фактора $g_{\|}=g_{e}, g_{\perp}=g_{e}(1-\lambda / \Delta E)$, где $g_{e}=2.0023$ - это $g$-фактор свободного электрона, $\lambda$ - константа спин-орбитального взаимодействия для С (отрицательная), и $\Delta E$ - это (положительная) разница в энергиях основного и возбужденного состояний. Предсказания таких расчетов, в частности, что $\Delta g_{\perp}>0$, находятся в качественном согласии с экспериментальными результатами. Конечно, для неаксиального дефекта $g$-тензор должен быть орторомбическим, но отклонение от цилиндрической симметрии крайне мало.

Таким образом, сделано заключение, что связь C-B - оборванная, бор находится в нейтральном состоянии и на нем нет значительной спиновой плотности. Нулевое отклонение $g_{z}$ от $g_{e}$ свидетельствует в пользу этой модели, в которой неспаренный электрон локализован на $p_{z}$-орбитали, потому что ожидаемая величина проекции орбитального углового момента на ось $z$ равна 
нулю. Полученные экспериментальные результаты показывают, что относительное геометрическое расположение главных осей тензоров $g$ и $A$ практически одинаково в аксиальном и неаксиальном мелких центрах В. Это подтверждает сходство между двумя конфигурациями мелкого бора.

Модель также объясняет противоположные знаки констант СТ структуры бора $A_{\|}$и $A_{\perp}(a$ и $b)$, которые наблюдались для $h$-позиции и $k$-узла в работах $[12,28]$. На боре нет значительной спиновой плотности и, следовательно, анизотропная часть СТ взаимодействия определяется диполь-дипольным взаимодействием с неспаренным спином на $p_{z}$-орбитали атома С. Это дипольдипольное взаимодействие может объяснить знак и порядок величины экспериментально наблюдаемых СТ параметров [23,25]. Во-первых, знак СТ константы $b$ должен быть положительным и СТ тензор аксиально симметричен с осью вдоль оборванной связи, в соответствии с экспериментальными данными. Для оценки величины $b$ применялось точечно-дипольное приближение. Оценка дает величину $b=+2.7 \mathrm{MHz}$ в хорошем соответствии с наблюдаемым значением порядка $+3 \mathrm{MHz}$.

Изотропная часть СТ взаимодействия бора очень мала и имеет отрицательный знак. Существует три механизма, которые могут привести к изотропному СТ взаимодействию с ядром, находящимся по соседству с парамагнитным дефектом. Это перекрытие орбиталей на различных центрах, ковалентность, обменная поляризация внутренних электронов. Первые два механизма дают положительную спиновую плотность, а третий механизм - отрицательную спиновую плотность на ядре. Последний механизм приводит к отрицательной спиновой плотности на ядре бора в мелких акцепторах бора из-за основного вклада прямой обменной поляризации. Наблюдаемая отрицательная спиновая плотность мелкого бора возникает из-за поляризации $s$-оболочки бора прямым взаимодействием с $p_{z}$-орбиталью иона С. Поляризация внутренних оболочек представляет собой разницу между плотностью электронов со спином вверх $(+)$ (параллельно неспаренному спину) и электронов со спином вниз $(-)$ на ядре. Следует понимать, что всего $0.1 \%$ спиновой плотности на $s$-орбитали бора дает $a \sim 3 \mathrm{MHz}$. Это указывает на существование тонкого баланса между различными типами поляризационных эффектов, который отвечает за величину $a$ и находится в соответствии с отсутствием значительной спиновой плотности на В. Значение $a-$ единственный параметр, который значительно отличается для гексагональных и квазикубических позиций акцептора, а также для акцепторов бора в разных политипах $\mathrm{SiC}$. Качественно эффекты поляризации могут быть объяснены на полуэмпирическом уровне для простых органических радикалов, но для кристаллов ситуация куда более сложная.

В отличие от мелких акцепторов бора, атомы $\mathrm{Al}$ и $\mathrm{Ga}$ занимают центральную замещающую атом $\mathrm{Si}$ позицию в решетке и, следовательно, наблюдается поведение, соответствующее теории эффективной массы.
Отметим, что для акцепторов Ве также были обнаружены свойства спектров ЭПР подобные мелким акцепторам бора [37]. Разница в ионных радиусах для замещающего кремний Ве и кремния составляет $\Delta R=-0.07 \AA$, что близко к соответствующему значению для бора, таким образом, Ве кажется хорошим кандидатом на нецентральное положение в решетке, как и бор.

Глубокие акцепторы. Особенности спектров ЭПР глубокого $\mathrm{Al}$ и глубокого Ga в основном совпадают с теми, которые наблюдались для глубокого бора.

1. Угловые зависимости спектров ЭПР показывают, что глубокие центры $\mathrm{B}, \mathrm{Al}, \mathrm{Ga}$ имеют одинаковую симметрию, близкую к аксиальной, относительно оси $c$ кристалла. Для глубоких акцепторов III группы не наблюдается поведения, описываемого в приближении эффективной массы.

2. При углах $\theta$ между $20^{\circ}$ и $60^{\circ}$ наблюдается разрешенная СТ структура спектров ЭПР.

3. Температурные зависимости спектров ЭПР показывают, что глубокие центры бора, галлия и алюминия демонстрируют схожее поведение при изменении температуры. В диапазоне температур $\sim 5-15 \mathrm{~K}$ происходят резкие изменения в спектрах ЭПР глубоких центров. В ориентациях, близких к $\mathbf{B} \| c, g$-фактор уменьшается и линии уширяются и исчезают при повышении температуры. Разрешение СТ структуры (для В и $\mathrm{Ga}$ ) также зависит от температуры и при повышении температуры выше $8 \mathrm{~K}$ для В и $6 \mathrm{~K}$ для $\mathrm{Ga}$ разрешенная структура начинает исчезать.

4. В ориентациях, близких к $\theta=0$, существует некоторый разброс значений $g$-факторов.

5. Обычно мелкие и глубокие акцепторы III группы наблюдаются одновременно. Соотношение их интенсивностей зависит от образца.

6. Константы СТ структуры в случае глубоких акцепторов близки по величине к константам в случае мелких акцепторов.

7. В параметрах спинового гамильтониана и температурных зависимостях спектров ЭПР для глубоких $\mathrm{B}, \mathrm{Al}$ и $\mathrm{Ga}$ наблюдаются только количественные различия.

Модели глубоких центров. Отметим, что независимо от размера примесного атома, входящего в состав глубокого центра, большего ( $\mathrm{Al}, \mathrm{Ga})$ или меньшего (В), чем кремний, физическое поведение центра и его микроскопическая модель одинаковы. Мы считаем, что модель глубокого бора, алюминия и галлия в акцепторном состоянии представляет собой пару элемент III группы-вакансия: $A_{\mathrm{Si}}-V_{\mathrm{C}}$, где $A=\mathrm{B}, \mathrm{Al}$ и Ga. $A_{\mathrm{Si}}$ - это B (Al, Ga), замещающий кремний, $V_{\mathrm{C}}$ - вакансия углерода (см. рис. 11, c). Аргументы в поддержку этой модели: центры имеют почти аксиальную симметрию относительно оси $c$ с сильной анизотропией, и СТ взаимодействие с ядрами В (Al, $\mathrm{Ga})$ имеет тот же порядок величины, что и для мелких акцепторных центров В (Al, Ga), представляющих собой атомы примеси, замещающие кремний. По-видимому, энергетически выгодно расположение вакансии углерода 
вдоль оси $с$ относительно примеси В (Al, Ga), замещающей кремний.

В качестве примера рассмотрим структуру глубокого акцептора бора [25] и распределение спиновой плотности, см. рис. 11, с. Предлагаемая модель глубокого бора предсказывает большое СТ расщепление для ядер ${ }^{29} \mathrm{Si}$, окружающих вакансию C, в то время как в спектрах ДЭЯР на рис. $3, b$ наблюдаются только линии ДЭЯР, расположенные близко к зеемановской частоте ${ }^{29} \mathrm{Si}$. Грубое приближение для СТ расщепления, ожидаемого для атомов ${ }^{29} \mathrm{Si}$, окружающих вакансию $\mathrm{C}$, может быть получено при рассмотрении расщепления в случае мелкого бора, где атом С несет основную спиновую плотность. На этом атоме углерода локализовано $38 \%$ спиновой плотности, и СТ взаимодействие с ним может быть разделено на изотропный вклад $a_{\mathrm{C}}=4.1 \mathrm{mT}$ и анизотропную часть $b_{\mathrm{C}}=1.1 \mathrm{mT}$. Предполагая, что на каждом из трех атомов $\mathrm{Si}$ спиновая плотность порядка 25-30\%, мы ожидаем СТ взаимодействие с ними того же порядка величины. Сателлитные линии в спектрах ЭПР $d \mathrm{~B}$ с СТ расщеплением порядка $4 \mathrm{mT}$ приписаны [24] атомам ${ }^{29} \mathrm{Si}$, окружающим вакансию, Si1 на рис. 11,c. Эти линии демонстрируют такую же температурную и угловую зависимости, как и основной сигнал глубокого бора.

Ядра $\mathrm{Si}$, обозначенные $\mathrm{Si1}$, по расчетам несут каждый спиновую плотность 25-30\% с изотропным СТ взаимодействием величиной примерно 3-4 $\mathrm{mT}$. На рис. 11, $\mathrm{B}(\mathrm{Si})$ обозначает положение примеси бора, замещающей $\mathrm{Si}$, a $V(\mathrm{C})$ обозначает положение вакансии углерода. На основании данных ДЭЯР и корреляционной спектроскопии сверхтонких подуровней (HYSCORE) предполагается следующее распределение спиновой плотности. На ядре бора спиновая плотность пренебрежимо мала. Примерно 25-30\% локализовано на каждом атоме Si1, а на каждом C1 локализовано порядка $\sim 0.2 \%$ спиновой плотности (2.2 MHz). На каждом С2 $\sim 0.8 \%(7.8 \mathrm{MHz})$, $\sim 0.2 \%$ на каждом $\mathrm{Si} 2$ вокруг $\mathrm{C} 1(2.6 \mathrm{MHz})$ и $\sim 0.1 \%$ на каждом Si2 вокруг C2. Другие атомы Si и C несут пренебрежимо малую спиновую плотность. Похожая модель предлагается для глубоких акцепторов алюминия и галлия.

Показано, что в отличие от мелких акцепторов бора, $g$-тензоры глубоких В (Al, Ga) практически аксиальны, a их главные оси $g_{z}$ параллельны оси $c$ как для гексагональной, так и для квазикубических позиций в решетке, при этом в спектрах ЭПР наблюдается небольшое отклонение от аксиальной симметрии (см. рис. 10,a). Кроме того, ширина линии ЭПР на частоте $95 \mathrm{GHz}$ примерно в десять раз больше, чем на частоте $9.3 \mathrm{GHz}$, это указывает на сильное уширение из-за напряжений в кристалле, приводящих к разбросу в $g$-факторах, что наиболее заметно в ориентациях, близких к В $\| c$. Рис. 11, $d$ иллюстрирует простое описание одноэлектронных линейных комбинаций атомных орбиталей - молекулярных орбиталей (LCAO-MO) для вакансии углерода рядом с бором, замещающим кремний [41]. Атомные орбитали представляют собой оборванные связи трех атомов $\mathrm{Si}(a, b$, и $c)$, окружающих вакансию. Точечная группа симметрии $C_{3 v}$ и полностью симметричная орбиталь $\left(a_{1}\right)$ ожидается в основном состоянии, а вырожденная орбиталь (e) лежит выше. Заполняя эти одноэлектронные орбитали неспаренными электронами трех атомов кремния, окружающих вакансию, можно видеть, что один электрон занимает орбиталь $e$, таким образом объясняя величину электронного спина $S=1 / 2$ дефекта. Из-за орбитального вырождения происходит статическое ян-теллеровское искажение, которое приводит к неэквивалентности одного из атомов $\mathrm{Si}$ по отношению к двум другим и к понижению симметрии до $C_{1 h}$. В результате орбиталь $e$ расщепляется на компоненты $a^{\prime}$ и $a^{\prime \prime}$ в соответствии с неприводимым представлением $C_{1 h}$. Наблюдаемые направления главных осей и величины главных значений $g$-тензора соответствуют этой модели. Ось $g_{z}$ направлена параллельно прямой бор-вакансия, а ось $g_{x}$ лежит в плоскости $\{11 \overline{2} 0\}$.

Для глубокого алюминия имеем $g_{\|}=2.28$ и $g_{\perp}=1.88$ при $4 \mathrm{~K}$. Очевидно отклонение от значения $g_{\perp}=0$, наблюдавшегося для мелкого A1. Для объяснения анизотропии $g$-факторов глубоких акцепторов III группы (Al, Ga) в $\mathrm{SiC}$ мы применим теоретическое рассмотрение, которое было развито для объяснения анизотропии $g$-факторов отрицательно заряженных центров кислорода ( $\mathrm{O}^{-}$центров) в перовскитах [42], характеризующихся дыркой в замкнутой $p$-оболочке кислорода. Было показано, что довольно слабое орторомбическое искажение решетки приводит к большому отклонению $g_{\perp}$ от нулевого значения и его сдвигу к значению $g_{S}=2$, что указывает на усиливающееся замораживание орбитального углового момента. Микроскопическая природа малой орторомбичности, необходимой для объяснения измеренных параметров $g$, обсуждалась выше и может быть следствием эффекта Яна-Теллера.

Рассмотрим $g$-факторы акцепторов Al, Ga, описываемых приближением эффективной массы, следуя работам $[43,44]$. Сначала мы проведем сравнительный анализ для кремния и $\mathrm{SiC}$. В кремнии минимальная энергия дырок наблюдается при $k=0$. Вырождение валентной зоны при $k=0$ связано с симметрией решетки и может быть снято (за исключением спинового вырождения) одноосным давлением. Энергии связи дырок с акцепторами III группы B, Al, Ga и In в $\mathrm{Si}$ находятся в интервале от примерно 0.05 до $0.16 \mathrm{eV}$. Поскольку спин-орбитальное расщепление полос $J=3 / 2$ и $1 / 2$ составляет $0.035 \mathrm{eV}$ [45], основное состояние четырехкратно вырождено. В работе [46] наблюдалась одна резонансная линия ЭПР в кристаллах легированных B, Al, Ga и In, подвергнутых одноосному сжатию. Эффективный $g$-фактор был представлен в виде $g^{2}=g_{\|}^{2} \cos ^{2} \theta+g_{\perp}^{2} \sin ^{2} \theta$, где $\theta-$ угол между осью давления (кубическое направление [001]) и магнитным полем. Спектры ЭПР акцепторов интерпретировались в терминах индуцированного давлением расщепления состояния $J=3 / 2$ на дублеты $M_{J}= \pm 1 / 2$ и $M_{J}= \pm 3 / 2$. 
Резонансный переход происходит между состояниями $M_{J}=-1 / 2$ и $M_{J}=+1 / 2$, которые расщепляются магнитным полем. Переход $M_{J}=-3 / 2 \leftrightarrow M_{J}=+3 / 2$ запрещен для большого расщепления в нулевом поле, переходы $M_{J}= \pm 1 / 2 \leftrightarrow M_{J}= \pm 3 / 2$ чувствительны к напряжениям в кристалле. Неудачи в обнаружении резонанса мелких акцепторов в отсутствие приложенного одноосного давления были связаны с уширением линий ЭПР случайными напряжениями и коротким временем спин-решеточной релаксации. Для свободной дырки в валентной зоне с $J=3 / 2$ теоретически $g_{\perp} \cong 2 g_{\|}$. Для мелкой дырки в $\mathrm{Si}$ наилучшее согласие с этим соотношением наблюдалось для акцепторов В, имеющих наименьшую энергию связи, что указывает на возможность применения приближения эффективной массы к этим дыркам. Экспериментально наблюдаемые особенности спектров ЭПР акцепторов $\mathrm{Al}, \mathrm{Ga}$ и In в $\mathrm{Si}$ в большинстве случаев идентичны тем, которые проявляются для В, количественные различия наблюдаются только в параметрах спинового гамильтониана.

В гексагональных политипах $\mathrm{SiC}(4 H-$ или $6 H-\mathrm{SiC})$ спектры ЭПР мелких акцепторов существенно отличаются от наблюдаемых для мелких акцепторов в кремнии. На рис. 12 (сверху) показана схема энергетических уровней для мелкого акцептора алюминия, с использованием результатов работ [42-44]. Расщепление уровней $\Delta$ в кристаллическом поле гексагонального кристалла $\mathrm{SiC}$ больше, чем спин-орбитальное расщепление $\lambda$, которое составляет около $7 \mathrm{meV}$ в $6 \mathrm{H}-\mathrm{SiC}$. Поэтому $\mathbf{J}=\mathbf{L}+\mathbf{S}$ уже не является хорошим квантовым числом. Самое верхнее состояние валентной зоны (основное состояние, соответствующее наименьшей энергии дырки) в представлении $\left|M_{J}\right\rangle\left|M_{S}\right\rangle$, имеет вид дублетов $|+1\rangle|\alpha\rangle,|-1\rangle|\beta\rangle$ и $|-1\rangle|\alpha\rangle,|+1\rangle|\beta\rangle$, где $| \pm 1\rangle$ состояния орбитального момента $L=1$, которые отщеплены от состояния $|0\rangle$ (дублет $|0\rangle|\alpha\rangle,|0\rangle|\beta\rangle$ ) гексагональным кристаллическим полем $\Delta$, a $|\alpha\rangle,|\beta\rangle-$ состояния со спином „вверх“ и „вниз“ соответственно. Неприводимое представление этого состояния - $\Gamma_{9}$ для кристаллической группы $C_{6 v}^{4}$ гексагонального политипа.

Эффект Зеемана для самого низкого дублета в основном состоянии описывается гамильтонианом

$$
\left.H_{Z e e}=\mu_{\mathrm{B}} \mathbf{H} \cdot\left(g_{L} \mathbf{L}+g\right)_{S} \mathbf{S}\right),
$$

где $L=1, S=1 / 2$. Здесь $g_{L}$ и $g_{S}-g$-факторы, связанные с орбитальным моментом и спином соответственно. Для $g \mu_{\mathrm{B}} H \ll \lambda, \Delta$ вырождение Крамерса снимается и вырожденные уровни расщепляются линейно по компоненте магнитного поля $H_{z}$ (ось $z$ параллельна оси $c \mathrm{SiC}$ ), что дает для самых низких состояний $\Delta E=\mu_{\mathrm{B}}\left(g_{S}+2 g_{L}\right) H_{Z}$, и в результате это приводит к зависимости $g(\theta)=g_{\|} \cos \theta$. Для случая свободной дырки в состоянии $\Gamma_{9}\left(g_{L}=1\right) g_{\|}=4.0$ и $g_{\perp}=0$. Наилучшее согласие с этим соотношением можно ожидать для акцепторов с наименьшей энергией связи, что было подтверждено экспериментально для $\mathrm{Al}$ и $\mathrm{Ga}$ в $\mathrm{SiC}$.

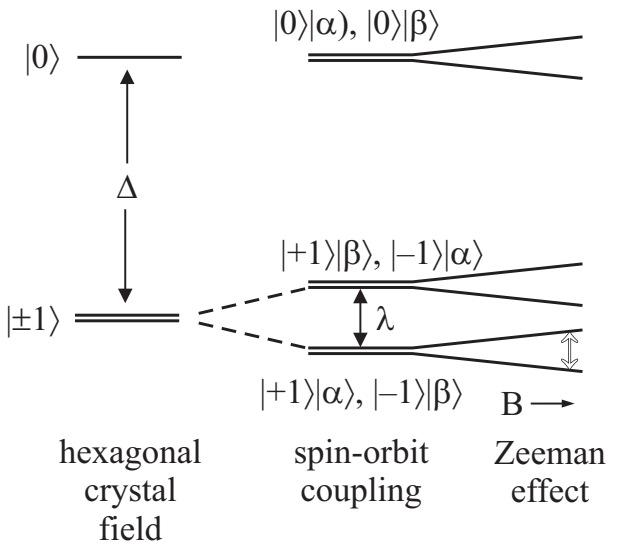

$a$

$b$

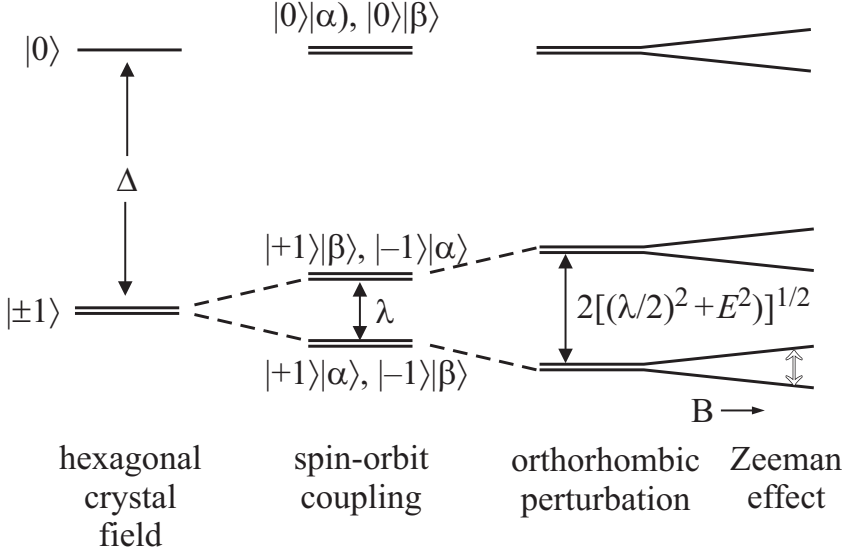

Рис. 12. Схема энергетических уровней акцепторных состояний в верхней части валентной зоны в гексагональном $\mathrm{SiC}$ при указанных возмущениях $(a)$. Орторомбическое возмущение приводит к дополнительному расщеплению уровней $(b)$.

Наблюдалось, что величина $g_{\|}$, равная 2.3-2.4 для мелкого акцептора $\mathrm{Al}$, уменьшается (приближается к 2) при увеличении энергии связи [18]. Угловая зависимость для $g$-фактора пропорциональна $\cos \theta$, что свидетельствует о применимости приближения эффективной массы для описания мелких акцепторов и отражает тот факт, что вершина валентной зоны находится в состоянии с симметрией $\Gamma_{9}$. При этом отмечается, что вклад орбитального углового момента уменьшается по сравнению со свободной дыркой вследствие локализации дырки в кристалле.

Возможным механизмом уменьшения орбитального момента является динамический эффект Яна-Теллера в случае, когда величина этого эффекта больше кристаллического поля. Влияние эффекта Яна-Теллера сильно зависит от степени локализации неспаренного электрона [18]. Эффект Яна-Теллера должен уменьшить спин-орбитальное расщепление между двумя дублетами $|+1\rangle|\alpha\rangle,|-1\rangle|\beta\rangle$ и $|-1\rangle|\alpha\rangle,|+1\rangle|\beta\rangle$ (рис. 12). Можно оценить эффективные значения спин-орбитального расщепления для мелкого акцептора $\mathrm{Al}$ в разных кристаллических позициях как $\lambda^{\prime} \cong g_{L}^{\prime} \lambda_{0}$, где для свободного 
экситона $\lambda_{0}, \sim 7 \mathrm{meV}: \lambda^{\prime}$ равно приблизительно $1.4 \mathrm{meV}$ и $1.1 \mathrm{meV}$.

В соответствии с [18,42-44], полный спиновый Гамильтониан имеет вид

$$
H_{S}=H_{a x}+H_{s o}+H_{o r t h}+H_{Z e e},
$$

где $H_{a x}=D\left(1-L_{z}^{2}\right), H_{s o}=\lambda \mathbf{L} \cdot \mathbf{S}, D-$ расщепление в нулевом магнитном поле.

В базисе $\left|M_{J}\right\rangle\left|M_{S}\right\rangle$ имеем $| \pm 1\rangle=(1 / \sqrt{2})\left(\left|p_{x}\right\rangle+i\left|p_{y}\right\rangle\right)$ и $|0\rangle=\left|p_{z}\right\rangle$. Член $H_{\text {orth }}=E\left(L_{x}^{2}-L_{y}^{2}\right)$ добавляется, чтобы учесть орторомбическое возмущение $(E-$ параметр орторомбического возмущения), характеризующее глубокие акцепторные центры, и приводит к расщеплению уровней, показанному на рис. 12, и к смешиванию состояний $|+1\rangle$ и $|-1\rangle$. Схема уровней и эффект Зеемана подобны схемам для $\mathrm{O}^{-}$центров в $\mathrm{KTaO}_{3}$ [44]. Следующие основные значения $g$ подлежат измерению для магнитного поля вдоль основных направлений, обозначенных нижними индексами:

$$
\begin{gathered}
g_{z}=g_{S}+2 g_{L} \sin v . \\
g_{x}=g_{S} \cos v-g_{L}(\lambda / D)(\cos v+1-\sin v), \\
g_{y}=g_{S} \cos v-g_{L}(\lambda / D)(\cos v+1+\sin v),
\end{gathered}
$$

где $\tan v=\lambda / 2 E$.

Оператор $L_{z}$ вносит вклад в первый порядок теории возмущений и приводит к выражению для $g_{z}$. Последний член в уравнениях для $g_{x}$ и $g_{y}$ умножается на малый множитель $\lambda / D$, который обусловлен вкладом операторов орбитального углового момента $L_{x}$ и $L_{y}$, входящих в гамильтониан зеемановского взаимодействия, связывающий состояния $|0\rangle$ и $|+1\rangle,|-1\rangle$. В случае аксиальной симметрии, когда $E=0$ и $v=\lambda / 2$, ожидаются следующие главные значения $g$-тензора: $g_{x}=g_{y}=g_{\perp}=0$ и $g_{z}=g_{\|}=4$ для $g_{L}=1$.

Измеренные значения явно отклоняются от этого предсказания. Однако небольшое орторомбическое возмущение, $E \neq 0$, а также отклонение $g$-фактора $g_{L}$ от единицы вследствие локализации дырки, могут объяснить экспериментальные данные. Как уже отмечалось, даже малое орторомбическое возмущение (малый параметр $E$ ) приводит к большому отклонению от $g_{\perp}=0$, что действительно наблюдалось для глубоких акцепторов алюминия и галлия. При этом $g_{\perp}$ сдвигается к $g_{S}=2.0$. Таким образом, наблюдаемые экспериментальные зависимости $g$-факторов глубоких акцепторов III группы в карбиде кремния свидетельствуют об отклонении от аксиальной симметрии и наличии орторомбических искажений. Эти искажения видны и в ориентационных зависимостях спектров ЭПР, представленных на рис. 10. Отклонение $g_{\|}$от теоретической величины 4 обусловлено гашением орбитального момента вследствие локализации дырки в валентной зоне карбида кремния.

Таким образом, B, Al и Ga формируют не только мелкие акцепторы, но также центры с глубокими акцепторными уровнями. Возможность формирования таких компенсирующих центров при высоких уровнях легирования $\mathrm{B}, \mathrm{Al}$ и $\mathrm{Ga}$ должна приниматься во внимание при создании материалов $p$-типа на основе карбида кремния.

\section{Список литературы}

[1] G. Pensl, R. Helbig, Festkoerperprobleme: Adv. Solid State Phys., 30 / Ed. U. Roessler. Vieweg, Braunschweig (1990). P. 133 and references therein.

[2] Г.А. Ломакин. ФТТ 7, 600 (1965).

[3] М.М. Аникин, А.А. Лебедев, А.Л. Сыркин, А.В. Суворов. ФТП 19, 69 (1985).

[4] H. Kuwabara, S. Yamada. Phys. Status Solidi A 30, 739 (1975).

[5] M. Ikeda, H. Matsunami, T. Tanaka. Phys. Rev. B 22, 2842 (1980).

[6] W. Suttrop, G. Pensl, P. Laning, Appl. Phys. A 51, 231 (1990).

[7] В.С. Балландович, Е.Н. Мохов. ФТП 29, 370 (1995).

[8] M. Ikeda, H. Matsunami, T. Tanaka. J. Lumin. 20, 111 (1979).

[9] H.H. Woodbury, G.W. Ludwig. Phys. Rev. 124, 1083 (1961).

[10] А.Г. Зубатов, И.М. Зарицкий, С.Н. Лукин, Е.Н. Мохов, В.Г. Степанов. ФТТ 27, 322 (1985).

[11] K. Maier, J. Schneider, W.Wilkening, S. Leibenzeder, R. Stein. Mater. Sci. Eng. B 11, 27 (1992).

[12] Н.П. Баран, В.Я. Братусь, А.А. Бугай, В.С. Сихнин, А.А. Климов, В.М. Максименко, Т.Л. Петренко, В.В. Романенко. ФТТ 35, 3135 (1993) и ссылки в ней.

[13] P.G. Baranov, V.A. Khramtsov, E.N. Mokhov. Semicond. Sci. Technol. 9, 1340 (1994).

[14] P.G. Baranov, E.N. Mokhov, Inst. Phys. Conf. Ser. 142, 293 (1996).

[15] P.G. Baranov, E.N. Mokhov. Semicond. Sci. Technol. 11, 489 (1996).

[16] P.G. Baranov, I.V. Ilyin, E.N. Mokhov. Solid State Communic. 100, 371 (1996).

[17] П.Г. Баранов, Е.Н. Мохов. ФТТ 38, 1446 (1996).

[18] K.M. Lee, Le Si Dang, G.D. Watkins, W.J. Choyke. Phys. Rev. B, 32, 2273 (1985).

[19] П.Г. Баранов, В.А. Ветров, Н.Г. Романов, В.И. Соколов. ФTT 27, 3459 (1985).

[20] P.G. Baranov, N.G. Romanov. Appl. Magn. Reson. 2, 361 (1991).

[21] P.G. Baranov, N.G. Romanov. Mater. Sci. Forum 83-87, 1207 (1992).

[22] P.G. Baranov. Defect and Diffusion Forum 148-149, 129 (1997).

[23] T. Matsumoto, O.G. Poluektov, J. Schmidt, E.N. Mokhov, P.G. Baranov. Phys. Rev. B 55, 2219 (1997).

[24] A.V. van Duijn-Arnold, T. Ikoma, O.G. Poluektov, P.G. Baranov, E.N. Mokhov, J. Schmidt. Phys. Rev. B, 57, 1607 (1998).

[25] A. van Duijn-Arnold, J. Mol, R. Verberk, J. Schmidt, E.N. Mokhov, P.G. Baranov. Phys. Rev. B 60, 15829 (1999).

[26] Т.Л. Петренко, В.В. Тесленко, Е.Н. Мохов. ФТП 26, 1556 (1992).

[27] T.L. Petrenko, A.A. Bugai, V.G. Baryakhtar, V.V. Teslenko, V.D. Khavryutchenko. Semicond. Sci. Technol. 9, 1849 (1994).

[28] R. Muller, M. Feege, S. Greulich-Weber, J.-M. Spaeth. Semicond. Sci. Technol. 8, 1377 (1993).

[29] J. Reinke, R. Muller, M. Feege, S. Greulich-Weber, J.-M. Spaeth. Mater. Sci. Forum 143-147, 63 (1994). 
[30] J. Reinke, S. Greulich-Weber, J.-M. Spaeth, E.N. Kalabukhova, S.N. Lukin, E.N. Mokhov. Inst. Phys. Conf. Ser. (UK) 137, 211 (1994).

[31] F.J. Adrian, S. Greulich-Weber, J.-M. Spaeth. Solid State Comm., 94, 41 (1995).

[32] Н.Г. Романов, В.А. Ветров, П.Г. Баранов, Е.Н. Мохов, В.Г. Одинг. Письма в ЖТФ 11, 1168 (1985).

[33] P.G. Baranov, N.G. Romanov, V.A. Vetrov, V.G. Oding. Proc. 20th Int. Conf. on the Physics of Semiconductors / Ed. E.M. Anastassakis, J.D. Joannopoulos. World Scientific, Dingapore (1990). V. 3. P. 1855.

[34] П.Г. Баранов, Е.Н. Мохов, А. Хофштеттер, А. Шарманн. Письма в ЖЭТФ 63, 803 (1996).

[35] B.K. Meyer, A. Hofstaetter, P.G. Baranov. Mater. Sci. Forum Vols 264-268, 591 (1998).

[36] J.R. Morton, K.F. Preston. J. Magn. Reson. 30, 577 (1978).

[37] A. van Duijn-Arnold, J. Schmidt, O.G. Poluektov, P.G. Baranov, E.N. Mokhov. Phys. Rev. B, 60, 15799 (1999).

[38] G. Bachelet, G.A. Baraff, M. Schulter, Phys. Rev. B 24, 4736 (1981).

[39] S.T. Pantelides, W.A. Harrison, F. Yndurain. Phys. Rev. B 34, 6038 (1986).

[40] H.B. Gray. Electrons and Chemical Bonding, W.A. Benjamin INC, N.Y.-Amsterdam (1965). 244 p.

[41] G.D. Watkins. Phys. Rev. 155, 802 (1967).

[42] M. Maiwald, 0.F. Schirmer. Europhys. Lett. 64, 776 (2003).

[43] Le Si Dang, R.M. Lee, G.D. Watkins. Phys. Rev. Lett. 45, 390 (1980).

[44] J.F. Scott, D.J. Toms, Le Si Dang, P.M. Lee, G.D. Watkins, W.J. Choyke. Phys. Rev. B 23, 2029 (1981).

[45] G.W. Ludwig, H.H. Woodbury. In: Solid State Physics. Vol. 13 / Ed. F. Seitz, D. Turnbull. Academic Press. N.Y. (1962). P. 233.

[46] G. Feher, J.C. Hensel, E.A. Gere. Phys. Rev. Lett. 5, 309 (1960). 